\title{
Confinement and moduli locking of Alice strings and monopoles
}

\author{
Muneto Nitta \\ Department of Physics \& Research and Education Center for Natural Sciences, Keio University, \\ Hiyoshi 4-1-1, Yokohama, Kanagawa 223-8521, Japan \\ E-mail: nitta@phys-h.keio.ac.jp
}

ABSTRACT: We argue that strings (vortices) and monopoles are confined, when fields receiving nontrivial Aharonov-Bohm (AB) phases around a string develop vacuum expectation values $(\mathrm{VEVs})$. We illustrate this in an $\mathrm{SU}(2) \times \mathrm{U}(1)$ gauge theory with charged triplet complex scalar fields admitting Alice strings and monopoles, by introducing charged doublet scalar fields receiving nontrivial $\mathrm{AB}$ phases around the Alice string. The Alice string carries a half $\mathrm{U}(1)$ magnetic flux and $1 / 4 \mathrm{SU}(2)$ magnetic flux taking a value in two of the $\mathrm{SU}(2)$ generators characterizing the $\mathrm{U}(1)$ modulus. This string is not confined in the absence of a doublet VEV in the sense that the $\mathrm{SU}(2)$ magnetic flux can be detected at large distance by an $\mathrm{AB}$ phase around the string. When the doublet field develops VEVs, there appear two kinds of phases that we call deconfined and confined phases. When a single Alice string is present in the deconfined phase, the $\mathrm{U}(1)$ modulus of the string and the vacuum moduli are locked (the bulk-soliton moduli locking). In the confined phase, the Alice string is inevitably attached by a domain wall that we call an $\mathrm{AB}$ defect and is confined with an anti-Alice string or another Alice string with the same $\mathrm{SU}(2)$ flux. Depending on the partner, the pair annihilates or forms a stable doubly-wound Alice string having an $\mathrm{SU}(2)$ magnetic flux inside the core, whose color cannot be detected at large distance by AB phases, implying the "color" confinement. The theory also admits stable Abrikosov-Nielsen-Olesen string and a $\mathbb{Z}_{2}$ string in the absence of the doublet VEVs, and each decays into two Alice strings in the presence of the doublet VEVs. A monopole in this theory can be constructed as a closed Alice string with the U(1) modulus twisted once, and we show that with the doublet VEVs, monopoles are also confined to monopole mesons of the monopole charge two.

Keywords: Solitons Monopoles and Instantons, Spontaneous Symmetry Breaking

ArXiv EPRINT: 2011.14396 


\section{Contents}

1 Introduction 1

2 The Alice theory 4

2.1 The model 4

2.2 Symmetry breaking by the triplet field 4

2.3 Symmetry breaking by the doublet field 6

2.4 The opposite hierarchy 8

3 Strings and monopoles $\quad 10$

$\begin{array}{lll}3.1 & \text { Abrikosov-Nielsen-Olesen string and } \mathbb{Z}_{2} \text { string } & 10\end{array}$

$\begin{array}{lll}3.2 & \text { Alice strings } & 11\end{array}$

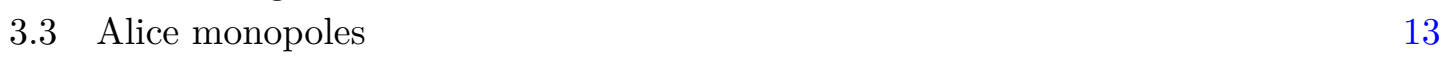

4 Confinement of Alice strings and monopoles $\quad \mathbf{1 5}$

$\begin{array}{lll}\text { 4.1 Aharonov-Bohm phases around an Alice string } & 15\end{array}$

$\begin{array}{lll}4.2 & \text { Bulk-soliton moduli locking } & 16\end{array}$

$\begin{array}{ll}4.3 \text { Aharonov-Bohm defects } & 17\end{array}$

$\begin{array}{ll}\text { 4.4 Classification of configurations of two strings } & 18\end{array}$

$\begin{array}{ll}\text { 4.5 Confinement of Alice strings } & 20\end{array}$

4.6 Decay of Abrikosov-Nielsen-Olesen string and $\mathbb{Z}_{2}$ string 22

4.7 Confinement of Alice monopoles: monopole mesons 23

$\begin{array}{llr}5 & \text { Summary and discussion } & 26\end{array}$

$\begin{array}{lr}\text { A Complex symmetric tensor } & 29\end{array}$

$\begin{array}{ll}\text { B Alice string configurations } & 30\end{array}$

\section{Introduction}

Color confinement is one of the most challenging problems in modern high energy physics. Particles transforming under color gauge group cannot be observed and are confined. Here, we discuss Aharonov-Bohm (AB) effects [1] may be one of key ingredients to understand color confinement. When a charged particle scatters from a solenoid with non-zero magnetic flux inside, a gauge potential rather than a field strength (a magnetic or electric field) yields an $\mathrm{AB}$ phase to the charged particle [1], resulting in a non-trivial differential scattering cross section. Such an AB effect was experimentally observed $[2,3]$. In the theory side, cosmic strings (vortices) exhibiting $\mathrm{AB}$ effects were proposed in cosmology [4-6] as well as in string theory [7-11]. Non-Abelian vortices in supersymmetric gauge theories [12-20] exhibit AB effects once a part of flavor symmetry is gauged [21-23]. In dense QCD, a color magnetic flux tube in the 2SC phase exhibits $\mathrm{AB}$ effects for quarks [24], and a non-Abelian vortex 
(color magnetic flux tube) in the color-flavor locked phase [25-30] exhibits (electromagnetic) $\mathrm{AB}$ effects for charged particles [31] as well as $\mathbb{Z}_{3}$ (color) $\mathrm{AB}$ effects for quarks [32-36]. NonAbelian Alice strings in two-flavor dense QCD exhibit nontrivial AB phases [37].

Alice strings are not only an example of AB strings but also exhibit a peculiar electromagnetic property. When electrically charged particles encircle around an Alice string, the signs of their electric charges are flipped because of non-single valuedness of an electromagnetic generator [38, 39]. The simplest Alice string is present in an $\mathrm{SO}(3)$ gauge theory with fiveplet scalar fields (real traceless symmetric tensor), where the $\mathrm{SO}(3)$ gauge symmetry is spontaneously broken down to $\mathrm{O}(2)$ identified with the electromagnetic $\mathrm{U}(1)$ group, which is not singly defined around the string [38, 40-43]. In quantum field theory, Alice strings were extensively studied for their exotic properties such as topological obstruction, non-local (Cheshire) charge, and non-Abelian statistics [40, 43-50]. In condensed matter physics, a global analogue of Alice strings was found in spinor Bose-Einstein condensates of ultracold atomic gases [51-54]. Recently, a $\mathrm{U}(1) \times \mathrm{SU}(2)$ (supersymmetric) gauge theory coupled with complex triplet scalar fields was found to admit a Bogomol'nyiPrasad-Sommerfield (BPS) [55, 56] Alice string [57-59]. ${ }^{1}$ The theory also admits a stable (BPS) Abrikosov-Nielsen-Olesen (ANO) string carrying a unit U(1) magnetic flux [63, 64], and a $\mathbb{Z}_{2}$ string connecting the two elements of the center of $\mathrm{SU}(2)$ and consequently carrying one half $\mathrm{SU}(2)$ magnetic flux [64]. ${ }^{2}$ In this case, the Alice string is a hybrid of these two kinds of vortices and carries one half U(1) magnetic flux of an ANO vortex and one quarter $\mathrm{SU}(2)$ magnetic flux taking a value in two of the $\mathrm{SU}(2)$ generators characterizing the U(1) modulus. On the other hand, a monopole in this theory can be constructed as a closed Alice string with the U(1) modulus twisted once, which was found in the global analogue [52]. Such a construction of monopole was also known in the conventional Alice theory, the $\mathrm{SO}(3)$ gauge theory with fiveplet scalars [40-43].

In the previous paper, in order to discuss what happens when a field receiving a non-trivial $\mathrm{AB}$ phase develops a vacuum expectation value (VEV), we further introduced doublet scalar fields receiving non-trivial $\mathrm{AB}$ phases in the presence of an Alice string, and found that a soliton or domain wall which we call an $\mathrm{AB}$ defect $^{3}$ is attached to the Alice string. ${ }^{4}$ This theory admits multiple BPS Alice strings at arbitrary positions, when we

\footnotetext{
${ }^{1}$ Actually, this model is a local (gauged) version of the theory admitting global Alice strings in refs. [51$54]$ in which the symmetry group $\mathrm{U}(1) \times \mathrm{SU}(2)$ is fully global. Recently, a hybrid of local and global Alice string was studied in the axion cosmology [60,61], in which the $\mathrm{U}(1)$ part is global and the $\mathrm{SU}(2)$ part is local. The opposite case in which the $\mathrm{U}(1)$ part is local and the $\mathrm{SU}(2)$ part is global is also known in certain superconductors [62].

${ }^{2}$ This is one half compared with the one generated by a closed loop in $\mathrm{SU}(2)$.

${ }^{3}$ Another example of an AB defect can be found in the Georgi-Machacek model [65] proposed as a model beyond the Standard Model (SM), consisting of three real triplet scalar fields and one doublet scalar field. If the triplet VEVs are larger than the doublet $\mathrm{VEV}$, then a $\mathbb{Z}_{2}$ string is attached by an $\mathrm{AB}$ defect [66].

${ }^{4}$ Although a string attached by an $\mathrm{AB}$ defect looks similar to a more conventional configuration of a string attached by a domain wall [67, 68], we emphasize that the mechanisms are rather different. For the conventional case, an explicit symmetry breaking term of the U(1) symmetry around which a string winds induces a domain wall. String-wall composites in axion models [72], two Higgs doublet models [73, $74]$, and domain wall skyrmions $[75,76]$ belong to this class. We may classify the two cases as follows; the conventional case is induced by "explicit symmetry breakings," while the AB defect is induced by "spontaneous symmetry breakings."
} 
turn off the doublet field. Since a doublet encircling two strings receives no AB phase, the two Alice strings should be connected by one AB defect when the doublet develops a VEV. One natural question was how each Alice string find a partner, when there are many Alice strings.

In this paper, we define the "color" (de)confinement as a situation that a color cannot (can) be detected at large distance by $\mathrm{AB}$ phases of some fields belonging to a representation of the color group. We then argue that the Alice string is not confined in the absence of a doublet VEV because the $\mathrm{SU}(2)$ (color) magnetic flux can be detected at large distance by an $\mathrm{AB}$ phase of the doublet fields around the string, while the confinement occurs once the doublet field develops VEVs (in the certain phase defined below). ${ }^{5}$ When the doublet field develops VEVs, there appear deconfined and confined phases with unbroken $\mathbb{Z}_{4}$ and $\mathbb{Z}_{2}$ symmetries, respectively. In the confined phase, the Alice string is inevitably attached by an AB defect and is confined with an anti-Alice string or another Alice string with the same $\mathrm{SU}(2)$ flux. Depending on the partner, the pair annihilates or forms a stable doubly-wound Alice string having an SU(2) magnetic flux inside the core, where the "color" cannot be seen at large distance by AB phases, implying the "color" confinement. We also show that with the doublet VEVs, monopoles are also confined to pair annihilate or to form monopole mesons of the monopole charge two. We thus call the unbroken phase without the doublet VEVs and the confined phase with the doublet VEVs as deconfined and confined phases, respectively.

Another interesting phenomenon is that in the presence of a single Alice string, the phase is enforced to be in the deconfined phase to annihilate an $\mathrm{AB}$ defect, and consequently the U(1) modulus of the string and the vacuum moduli of the doublet fields are locked. We call this phenomenon as the bulk-soliton moduli locking. We also show that while an ANO string and a $\mathbb{Z}_{2}$ string are stable in the absence of the doublet VEVs, each decays into two Alice strings to each of which an $\mathrm{AB}$ defect is attached, once the doublet field develops the VEVs. We discuss that whether a set of two strings exhibits confinement or decay can be determined from $\mathrm{AB}$ phases of the doublet field encircling the two strings.

This paper is organized as follows. In section 2, we introduce our model admitting BPS Alice strings and discuss in detail symmetry breaking patterns when the triplet field develops VEVs at high energy and the doublet field also develops VEVs at low energy. We also discuss the opposite hierarchy in which the doublet field develops VEVs at high energy and the triplet field develops VEVs at low energy. In section 3, we introduce an ANO string, a $\mathbb{Z}_{2}$ string, an Alice string, and an Alice monopole. In section 4, we first discuss $\mathrm{AB}$ phases of the doublet fields around a single Alice string and around two strings. We then discuss, in the presence of doublet VEVs, the bulk-soliton moduli locking, confinement of Alice strings and monopoles, and decay of ANO and $\mathbb{Z}_{2}$ strings. Section 5 is devoted to a summary and discussion. In appendix A, we show that complex adjoint scalar fields are equivalent to complex $2 \times 2$ symmetric tensor scalar fields. In appendix B, we give a brief review of previous results for BPS Alice string solution [57, 58] and an AB defect [59].

\footnotetext{
${ }^{5}$ In this definition, although a non-Abelian vortex in the CFL phase of dense QCD carries a color magnetic flux [25-30], it exhibits only a $\mathbb{Z}_{3} \mathrm{AB}$ phase within $\mathrm{SU}(3)$ color symmetry, which is color singlet. Namely, a color flux of the non-Abelian vortex cannot be seen from large distance, and consequently it is already confined as it is. The same holds for non-Abelian vortices in supersymmetric gauge theories [12-20] unless a part of flavor symmetry is gauged [21-23].
} 


\section{The Alice theory}

\section{$2.1 \quad$ The model}

We consider an $G=\mathrm{SU}(2) \times \mathrm{U}(1)$ gauge theory coupled with one charged complex triplet (adjoint) scalar field $\Phi=\sum_{\alpha=1}^{3} \Phi^{\alpha} \sigma^{\alpha}$ and one (charged) doublet scalar field $\Psi$. Since the matter content is the same with the gauge and Higgs sectors of the triplet Higgs model beyond the SM, we label the gauge group as $G=\mathrm{SU}(2)_{W} \times \mathrm{U}(1)_{Y} \cdot{ }^{6}$ The $\mathrm{U}(1)_{Y}$ charge of the triplet is $Y=1$, while the doublet fields $q\left(q=\frac{1}{2}\right.$ is the choice of the triplet Higgs model). In the previous paper [59], we considered the cases $q=\frac{1}{2}$ and $q=0$, but here we mostly consider the former.

The Lagrangian is given by

$$
\mathcal{L}=-\frac{1}{4} \operatorname{Tr} F_{\mu \nu} F^{\mu \nu}-\frac{1}{4} f_{\mu \nu} f^{\mu \nu}+\operatorname{Tr}\left|D_{\mu} \Phi\right|^{2}+\left|\mathcal{D}_{\mu} \Psi\right|^{2}-V(\Phi, \Psi)
$$

where $\mathcal{D}_{\mu} \Phi^{\alpha}=\partial_{\mu} \Phi^{\alpha}-i e a_{\mu} \Phi+g \epsilon^{\alpha \beta \gamma} A_{\mu}^{\beta} \Phi^{\gamma}, \mathcal{D}_{\mu} \Psi=\partial_{\mu} \Psi-i q a_{\mu} \Psi-i A_{\mu} \Psi, F_{\mu \nu}=\partial_{\mu} A_{\nu}-$ $\partial_{\nu} A_{\mu}-i g\left[A_{\mu}, A_{\nu}\right], f_{\mu \nu}=\partial_{\mu} a_{\nu}-\partial_{\nu} a_{\mu}$. Here $e$ and $g$ are the coupling constants of the $\mathrm{U}(1)_{Y}$ and $\mathrm{SU}(2)_{W}$ gauge fields, respectively. The potential term is given by

$$
V(\Phi, \Psi)=V_{\Phi}(\Phi)+V_{\Psi}(\Psi)+V_{\mathrm{int}}(\Phi, \Psi)
$$

with

$$
\begin{aligned}
V_{\Phi}(\Phi) & =\frac{\lambda_{g}}{4} \operatorname{Tr}\left[\Phi, \Phi^{\dagger}\right]^{2}+\frac{\lambda_{e}}{2}\left(\operatorname{Tr} \Phi \Phi^{\dagger}-2 \xi^{2}\right)^{2}, \\
V_{\Psi}(\Psi) & =M^{2} \Psi^{\dagger} \Psi+\lambda_{\psi}\left(\Psi^{\dagger} \Psi\right)^{2}, \\
V_{\text {int }}(\Phi, \Psi) & =\mu\left(\Psi_{c}^{\dagger} \Phi^{*} \Psi+\Psi^{\dagger} \Phi \Psi_{c}\right)+\lambda_{1} \Psi^{\dagger} \Psi \operatorname{Tr}\left(\Phi^{\dagger} \Phi\right)+\lambda_{2} \Psi^{\dagger} \Phi^{\dagger} \Phi \Psi,
\end{aligned}
$$

where $\lambda_{g}$ and $\lambda_{e}$ are two couplings of the triplet, $\xi$ is a parameter giving a VEV of the triplet, $M$ is the bare mass of the doublet, $\lambda_{\psi}$ is the quartic coupling of the doublet field, and $\mu, \lambda_{1}, \lambda_{2}$ are couplings between the doublet and triplet scalar fields. The charge conjugation of the doublet field is defined as $\Psi_{c}=i \sigma^{2} \Psi^{*}$.

Before discussing details in the next section, here let us summarize the symmetry breaking patterns in figure 1 and vacuum manifolds and corresponding lower dimensional homotopy groups in table 1 .

\subsection{Symmetry breaking by the triplet field}

First, let us turn off the doublet field and consider the triplet field. We choose the vacuum expectation value of the triplet field $\Phi$ without loss of generality as

$$
\langle\Phi\rangle=\xi \sigma^{1} .
$$

This triplet VEV breaks the gauge symmetry group spontaneously as ${ }^{7}$

$$
G=\mathrm{U}(1)_{Y} \times \mathrm{SU}(2)_{W} \longrightarrow H=\left(\mathbb{Z}_{4}\right)_{Y+W_{2,3}} \ltimes \mathrm{U}(1)_{W_{1}} .
$$

\footnotetext{
${ }^{6}$ However we consider a different phase and different parameter region.

${ }^{7}$ When we do not have a doublet field, the actual gauge group is $\mathrm{SU}(2) / \mathbb{Z}_{2}=\mathrm{SO}(3)$ since the center $\mathbb{Z}_{2}$ does not act on the triplet. Even in this case, it is useful to consider the universal covering $\mathrm{SU}(2)$ for the symmetry breaking.
} 


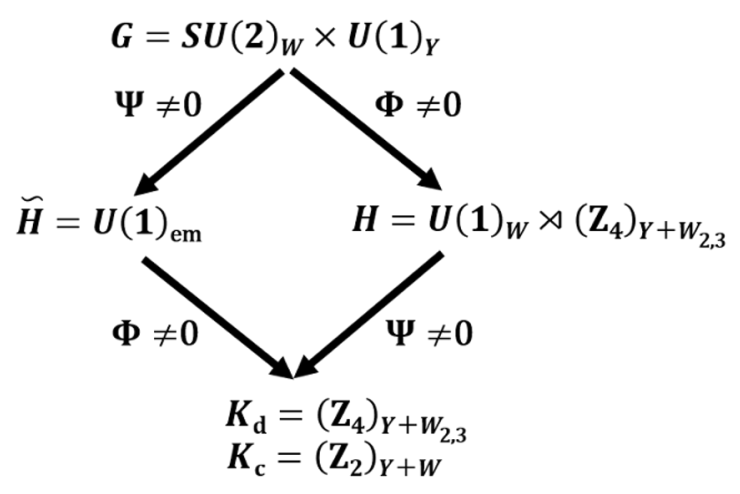

Figure 1. Spontaneous symmetry breaking patterns.

\begin{tabular}{|c|c|c|c|c|}
\hline Vacuum manifolds & $\pi_{0}$ & $\pi_{1}$ & $\pi_{2}$ & $\pi_{3}$ \\
\hline$\frac{G}{H}=\frac{\mathrm{U}(1)_{Y} \times \mathrm{SU}(2)_{W}}{\left(\mathbb{Z}_{4}\right)_{Y+W_{2,3}} \ltimes \mathrm{U}(1)_{W_{1}}} \simeq \frac{S_{Y}^{1} \times S_{W}^{2}}{\left(\mathbb{Z}_{2}\right)_{Y+W_{2,3}}}$ & & $\begin{array}{c}\mathbb{Z} \\
\text { Alice string }\end{array}$ & $\begin{array}{c}\mathbb{Z} \\
\text { monopole }\end{array}$ & $\mathbb{Z}$ \\
\hline$\frac{H}{K_{\mathrm{d}}} \simeq S^{1}$ & & $\begin{array}{c}\mathbb{Z} \\
\text { Another string }\end{array}$ & & \\
\hline$\frac{H}{K_{\mathrm{c}}} \simeq\left(\mathbb{Z}_{2}\right)_{Y+W_{2,3}} \ltimes \mathrm{U}(1)_{W_{1}}$ & $\begin{array}{c}\mathbb{Z}_{2} \\
\text { wall }\end{array}$ & $\begin{array}{c}\mathbb{Z} \\
\text { Another string }\end{array}$ & & \\
\hline$\frac{G}{\tilde{H}}=\frac{\mathrm{U}(1)_{Y} \times \mathrm{SU}(2)_{W}}{\mathrm{U}(1)_{Y+W_{3}}} \simeq S^{3}$ & & & & $\mathbb{Z}$ \\
\hline$\frac{\tilde{H}}{K_{\mathrm{d}}}=\frac{\mathrm{U}(1)_{Y+W_{3}}}{\left(\mathbb{Z}_{4}\right)_{Y+W_{3}}}$ & & $\begin{array}{c}\mathbb{Z} \\
1 / 4 \text { Abelian string }\end{array}$ & & \\
\hline$\frac{\tilde{H}}{K_{\mathrm{c}}}=\frac{\mathrm{U}(1)_{Y+W_{3}}}{\left(\mathbb{Z}_{2}\right)_{Y+W}}$ & & $\begin{array}{c}\mathbb{Z} \\
1 / 2 \text { Abelian string }\end{array}$ & & \\
\hline$\frac{G}{K_{\mathrm{d}}}=\frac{\mathrm{U}(1)_{Y} \times \mathrm{SU}(2)_{W}}{\left(\mathbb{Z}_{4}\right)_{Y+W_{2,3}}} \simeq \frac{\mathrm{U}(2)_{Y+W}}{\left(\mathbb{Z}_{2}\right)_{Y+W_{2,3}}}$ & & $\begin{array}{c}\mathbb{Z} \\
1 / 4 \text { Abelian string }\end{array}$ & & $\mathbb{Z}$ \\
\hline$\frac{G}{K_{\mathrm{c}}}=\frac{\mathrm{U}(1)_{Y} \times \mathrm{SU}(2)_{W}}{\left(\mathbb{Z}_{2}\right)_{Y+W}} \simeq \mathrm{U}(2)_{Y+W}$ & & $\begin{array}{c}\mathbb{Z} \\
1 / 2 \text { Abelian string }\end{array}$ & & $\mathbb{Z}$ \\
\hline
\end{tabular}

Table 1. Vacuum manifolds and corresponding lower dimensional homotopy groups. 
Here, $\mathrm{U}(1)_{W_{1}}$ is generated by $\sigma^{1}$, and $\ltimes$ denotes a semi-direct product implying that the $\mathrm{U}(1)_{W_{1}}$ acts on the $\left(\mathbb{Z}_{4}\right)_{Y+W_{2,3}}$ symmetry. The $\left(\mathbb{Z}_{4}\right)_{Y+W_{2,3}}$ symmetry is defined by a simultaneous action of a $\pi$ rotation of the $\mathrm{U}(1)_{Y}$ group and an $\mathrm{SU}(2)_{W}$ element generated by a linear combination of $\sigma^{2}$ and $\sigma^{3}$,

$$
e^{i \frac{\pi}{2} \mathbf{n} \cdot \vec{\sigma}}=\left(\begin{array}{cc}
i \cos \beta & \sin \beta \\
-\sin \beta & -i \cos \beta
\end{array}\right), \quad \mathbf{n}=(0, \sin \beta, \cos \beta),
$$

i.e., the minimum element of $\left(\mathbb{Z}_{4}\right)_{Y+W_{2,3}}$ is given by

$$
\left(\mathbb{Z}_{4}\right)_{Y+W_{2,3}}: \quad\left(e^{i \pi}, e^{i \frac{\pi}{2} \mathbf{n} \cdot \vec{\sigma}}\right) \in \mathrm{U}(1)_{Y} \times \mathrm{SU}(2)_{W}: \quad e^{i \pi} e^{i \frac{\pi}{2} \mathbf{n} \cdot \vec{\sigma}} \sigma^{1} e^{-i \frac{\pi}{2} \mathbf{n} \cdot \vec{\sigma}}=\sigma^{1} .
$$

Because of $\left(e^{i \pi}\right)^{2}=1$ and $\left(e^{i \frac{\pi}{2} \mathbf{n} \cdot \vec{\sigma}}\right)^{2}=-\mathbf{1}_{2}$, the double action in eq. (2.9),

$$
\left(\mathbb{Z}_{2}\right)_{Y+W} \text { on } \Phi:\left(1,-\mathbf{1}_{2}\right) \in \mathrm{U}(1)_{Y} \times \mathrm{SU}(2)_{W},
$$

is an element of the center of $\mathrm{SU}(2)_{W} \cdot{ }^{8}$ We thus have a relation

$$
\left(\mathbb{Z}_{4}\right)_{Y+W_{2,3}}=\left(\mathbb{Z}_{2}\right)_{Y+W_{2,3}} \times\left(\mathbb{Z}_{2}\right)_{Y+W} .
$$

By noting $\mathrm{SU}(2)_{W} /\left(\mathbb{Z}_{2}\right)_{Y+W}=\mathrm{SO}(3)_{W}$ with the footnote 8 , the vacuum manifold is found to be

$$
\frac{G}{H}=\frac{\mathrm{U}(1)_{Y} \times \mathrm{SU}(2)_{W}}{\left(\mathbb{Z}_{4}\right)_{Y+W_{2,3}} \ltimes \mathrm{U}(1)_{W_{1}}} \simeq \frac{\mathrm{U}(1)_{Y} \times\left[\mathrm{SU}(2)_{W} /\left(\mathbb{Z}_{2}\right)_{Y+W}\right]}{\left(\mathbb{Z}_{2}\right)_{Y+W_{2,3}} \ltimes \mathrm{U}(1)_{W_{1}}} \simeq \frac{S_{Y}^{1} \times S_{W}^{2}}{\left(\mathbb{Z}_{2}\right)_{Y+W_{2,3}}} .
$$

In the rightmost expression, the $\left(\mathbb{Z}_{2}\right)_{Y+W_{2,3}}$ symmetry is generated by the first factor of the right hand side of eq. (2.11).

The lower dimensional homotopy groups for this manifold can be calculated as

$$
\pi_{0}\left(\frac{G}{H}\right) \simeq\{0\}, \quad \pi_{1}\left(\frac{G}{H}\right) \simeq \mathbb{Z}, \quad \pi_{2}\left(\frac{G}{H}\right) \simeq \mathbb{Z}, \quad \pi_{3}\left(\frac{G}{H}\right) \simeq \mathbb{Z},
$$

indicating the absence of domain walls, the existence of stable strings (vortices), and monopoles. ${ }^{9}$

\subsection{Symmetry breaking by the doublet field}

Next, let us turn on the doublet field supposing that the triplet field develops VEVs at high energy and the doublet develops VEVs at low energy. The $\mathrm{U}(1)_{W_{1}}$ group in $H$ in eq. (2.7) must be spontaneously broken by the doublet VEVs, but the breaking pattern of the $\left(\mathbb{Z}_{4}\right)_{Y+W_{2,3}}$ symmetry depends on the VEVs. The $\left(\mathbb{Z}_{4}\right)_{Y+W_{2,3}}$ symmetry in eq. (2.9) acts on the doublet field $\Psi=(a, b)^{T}\left(|a|^{2}+|b|^{2}=v^{2}\right)$ with the $\mathrm{U}(1)_{Y}$ charge $q$ as

$$
\Psi^{\prime}=e^{i \pi q}\left(\begin{array}{cc}
i \cos \beta & \sin \beta \\
-\sin \beta & -i \cos \beta
\end{array}\right)\left(\begin{array}{l}
a \\
b
\end{array}\right)=e^{i \pi q}\left(\begin{array}{c}
i a \cos \beta+b \sin \beta \\
-i b \cos \beta-a \sin \beta
\end{array}\right) .
$$

From now on, we consider the case of $q=1 / 2$.

\footnotetext{
${ }^{8}$ One has to be careful about the fact that this action behaves as a center only on the triplet field. If there is another field with a non-integer $\mathrm{U}(1)_{Y}$ charge, a non-trivial action of the $\mathrm{U}(1)_{Y}$ action remains on such a field. Our doublet field is such a field, see eq. (2.18), below. Thus, we write it as $\left(\mathbb{Z}_{2}\right)_{Y+W}$ with " $Y+$."

${ }^{9}$ From the nontrivial third homotopy group $\pi_{3}$, Hopfions are possible at least for global analogues [78], but it is unclear whether gauged Hopfions stably exist for the local case.
} 
We call the case in which the $\left(\mathbb{Z}_{4}\right)_{Y+W_{2,3}}$ symmetry in eq. (2.9) is unbroken as the deconfined phase, and otherwise the confined phase. Two typical solutions of the deconfined phase are

$$
\left\{\begin{array}{l}
\Psi=\left(\begin{array}{l}
0 \\
v
\end{array}\right), \cos \beta=+1, K=\left(\mathbb{Z}_{4}\right)_{Y+W_{3}} \\
\Psi=\left(\begin{array}{l}
v \\
0
\end{array}\right), \cos \beta=-1, K=\left(\mathbb{Z}_{4}\right)_{Y-W_{3}},
\end{array}\right.
$$

where $\left(\mathbb{Z}_{4}\right)_{Y+W_{2,3}}$ in eqs. (2.8) and (2.9) is generated by a $\pi$ rotation of $\mathrm{U}(1)$ and $+\sigma^{3}$ or $-\sigma^{3}$ in the $\mathrm{SU}(2)$ group, respectively, and so we have denoted it by $\left(\mathbb{Z}_{4}\right)_{Y \pm W_{3}}$. More general solution of the deconfined phase is found to be

$$
\Psi=U\left(\begin{array}{l}
0 \\
v
\end{array}\right)=\left(\begin{array}{c}
i v \sin \frac{\beta}{2} \\
v \cos \frac{\beta}{2}
\end{array}\right), \quad K=\left(\mathbb{Z}_{4}\right)_{Y+W_{2,3}},
$$

with $\left(\mathbb{Z}_{4}\right)_{Y+W_{2,3}}$ in eq. (2.9) and $U$ defined by

$$
U=e^{\frac{i}{2} \beta \sigma^{1}}=\cos \frac{\beta}{2} \mathbf{1}_{2}+i \sigma^{1} \sin \frac{\beta}{2}=\left(\begin{array}{cc}
\cos \frac{\beta}{2} & i \sin \frac{\beta}{2} \\
i \sin \frac{\beta}{2} & \cos \frac{\beta}{2}
\end{array}\right) .
$$

Here, $\beta=0$ and $\beta=\pi$ correspond to the two cases in eq. (2.15) (up to the constant factor $i)$, respectively. The parameter $\beta$ corresponds to the $\mathrm{U}(1)_{W_{1}}$ (would-be) Nambu-Goldstone mode associated with the $\mathrm{U}(1)_{W_{1}}$ symmetry spontaneously broken by the doublet.

From the $\left(\mathbb{Z}_{4}\right)_{Y+W_{2,3}}$ action on $\Psi$ in eq. (2.14) with $q=1 / 2$, the double action,

$$
\left(\mathbb{Z}_{2}\right)_{Y+W} \text { on } \Psi:\left(-1,-\mathbf{1}_{2}\right) \in \mathrm{U}(1)_{Y} \times \mathrm{SU}(2)_{W},
$$

always leaves arbtrary $\Psi$ invariant, thanks to the particular choice of $q=1 / 2$. As noted in footnote 8 , this $\left(\mathbb{Z}_{2}\right)_{Y+W}$ action behaves as a center of $\mathrm{SU}(2)_{W}$ when acting only on the triplet field $\Phi$ as in eq. (2.10) but it is not so on the doublet field $\Psi$ because of the -1 element of $\mathrm{U}(1)_{Y}$. This fact becomes important in the vacuum manifolds. Thus, in the confined phase, the $\left(\mathbb{Z}_{4}\right)_{Y+W_{2,3}}$ is spontaneously broken to a $\left(\mathbb{Z}_{2}\right)_{Y+W}$ subgroup. ${ }^{10}$

One can easily verify that the configurations in eq. (2.16) satisfy the gauge invariant (covariant) condition

$$
\Psi^{\dagger} \Phi \Psi=0
$$

Thus, the phases and unbroken symmetries can be summarized as

$$
\begin{array}{rlrl}
\text { deconfined phase: } & \Psi^{\dagger} \Phi \Psi=0, & K_{\mathrm{d}} & =\left(\mathbb{Z}_{4}\right)_{Y+W_{2,3}}, \\
\text { confined phase: } & \left|\Psi^{\dagger} \Phi \Psi\right| \neq 0, & K_{\mathrm{c}}=\left(\mathbb{Z}_{2}\right)_{Y+W},
\end{array}
$$

for the deconfined and confined phases, respectively.

\footnotetext{
${ }^{10}$ For different choice of $q$, this $\left(\mathbb{Z}_{2}\right)_{Y+W}$ is broken in general.
} 
The vacuum manifolds for the second symmetry breaking are

$$
\begin{aligned}
\text { deconfined phase: } & \frac{H}{K_{\mathrm{d}}} & =S^{1}, \\
\text { confined phase: } & \frac{H}{K_{\mathrm{c}}} & =\left(\mathbb{Z}_{2}\right)_{Y+W_{2,3}} \ltimes \mathrm{U}(1)_{W_{1}} .
\end{aligned}
$$

For the both cases, the $S^{1} \simeq \mathrm{U}(1)_{W_{1}}$ part of the vacuum manifolds is parameterized by $\beta$ in eq. (2.16). The associated lower dimensional homotopy groups for the second symmetry breaking are

$$
\begin{aligned}
\text { deconfined : } & \pi_{0}\left(\frac{H}{K_{\mathrm{d}}}\right) \simeq\{0\}, \quad \pi_{1}\left(\frac{H}{K_{\mathrm{d}}}\right) \simeq \mathbb{Z}, \quad \pi_{2}\left(\frac{H}{K_{\mathrm{d}}}\right) \simeq\{0\}, \quad \pi_{3}\left(\frac{H}{K_{\mathrm{d}}}\right) \simeq\{0\}, \\
\text { confined : } & \pi_{0}\left(\frac{H}{K_{\mathrm{c}}}\right) \simeq \mathbb{Z}_{2}, \quad \pi_{1}\left(\frac{H}{K_{\mathrm{c}}}\right) \simeq \mathbb{Z}, \quad \pi_{2}\left(\frac{H}{K_{\mathrm{c}}}\right) \simeq\{0\}, \quad \pi_{3}\left(\frac{H}{K_{\mathrm{c}}}\right) \simeq\{0\} .
\end{aligned}
$$

These show that both the phases allow another type of vortices, and that the confined phase allows $\mathbb{Z}_{2}$ domain walls while the deconfined phase does not.

Finally, the vacuum manifolds of the overall breaking by the both triplet and doublet fields are

$$
\begin{array}{rlrl} 
& \text { deconfined phase: } & \frac{G}{K_{\mathrm{d}}} & =\frac{\mathrm{U}(1)_{Y} \times \mathrm{SU}(2)_{W}}{\left(\mathbb{Z}_{4}\right)_{Y+W_{2,3}}} \simeq \frac{\left(\frac{\mathrm{U}(1)_{Y} \times \mathrm{SU}(2)_{W}}{\left(\mathbb{Z}_{2}\right)_{Y+W}}\right)}{\left(\mathbb{Z}_{2}\right)_{Y+W_{2,3}}} \simeq \frac{\mathrm{U}(2)_{Y+W}}{\left(\mathbb{Z}_{2}\right)_{Y+W_{2,3}}}, \\
\text { confined phase: } & \frac{G}{K_{\mathrm{c}}} & =\frac{\mathrm{U}(1)_{Y} \times \mathrm{SU}(2)_{W}}{\left(\mathbb{Z}_{2}\right)_{Y+W}} \simeq \mathrm{U}(2)_{Y+W},
\end{array}
$$

for the deconfined and confined phases, respectively. ${ }^{11}$ The lower dimensional homotopy groups are

$$
\begin{array}{rlrl}
\text { deconfined: } & \pi_{0}\left(\frac{G}{K_{\mathrm{d}}}\right) \simeq\{0\}, & \pi_{1}\left(\frac{G}{K_{\mathrm{d}}}\right) \simeq \mathbb{Z}, \quad \pi_{2}\left(\frac{G}{K_{\mathrm{d}}}\right) \simeq\{0\}, \quad \pi_{3}\left(\frac{G}{K_{\mathrm{d}}}\right) \simeq \mathbb{Z}, \\
\text { confined: } & \pi_{0}\left(\frac{G}{K_{\mathrm{c}}}\right) \simeq\{0\}, \quad \pi_{1}\left(\frac{G}{K_{\mathrm{c}}}\right) \simeq \mathbb{Z}, \quad \pi_{2}\left(\frac{G}{K_{\mathrm{c}}}\right) \simeq\{0\}, \quad \pi_{3}\left(\frac{G}{K_{\mathrm{c}}}\right) \simeq \mathbb{Z} .
\end{array}
$$

The both phases have nontrivial first homotopy groups. However, the minimal elements are different as it will become important later.

For the case of $q=0$, the condition $\Psi^{\prime}=\Psi=(a, b)^{T}$ with eq. (2.14) cannot be satisfied, implying that the $\mathbb{Z}_{4}$ symmetry is broken completely by the VEV of the doublet (where no $\mathbb{Z}_{2}$ remians).

\subsection{The opposite hierarchy}

Although we mainly consider the case that the triplet VEVs are much greater than the doublet VEVs, let us briefly mention the opposite case (with the doublet $\mathrm{U}(1)_{Y}$ charge

\footnotetext{
${ }^{11}$ It is interesting to note that, eventually, the vacuum manifold of the overall breaking in the confined phase coincides with that of the U(2) Higgs model admitting non-Abelian vortices [12-20].
} 
$q=1 / 2$ ), in which the doublet VEVs are much greater than the triplet VEVs. As the case of the SM, when the doublet field develops VEVs, we can take

$$
\Psi=(v, 0)^{T}
$$

without loss of generality. The symmetry is broken as ${ }^{12}$

$$
G=\mathrm{U}(1)_{Y} \times \mathrm{SU}(2)_{W} \longrightarrow \tilde{H}=\mathrm{U}(1)_{Y+W_{3}}\left[=\mathrm{U}(1)_{\mathrm{em}}\right]
$$

where $\mathrm{U}(1)_{Y+W_{3}}$ is defined by

$$
\mathrm{U}(1)_{Y+W_{3}}: \Psi \rightarrow e^{-i \alpha} e^{i \alpha \sigma^{3}} \Psi
$$

which is the electromagnetic $\mathrm{U}(1)_{\mathrm{em}}$ in the context of the SM. The vacuum manifold is

$$
\frac{G}{\tilde{H}}=\frac{\mathrm{U}(1)_{Y} \times \mathrm{SU}(2)_{W}}{\mathrm{U}(1)_{Y+W_{3}}} \simeq \mathrm{SU}(2) \simeq S^{3},
$$

with lower dimensional homotopy groups

$$
\pi_{0}\left(\frac{G}{\tilde{H}}\right) \simeq\{0\}, \quad \pi_{1}\left(\frac{G}{\tilde{H}}\right) \simeq\{0\}, \quad \pi_{2}\left(\frac{G}{\tilde{H}}\right) \simeq\{0\}, \quad \pi_{3}\left(\frac{G}{\tilde{H}}\right) \simeq \mathbb{Z},
$$

which are topologically almost trivial as is well known.

Then, when the triplet develops VEVs at low energy, there are two phases as summarized in eq. (2.20):

$$
\begin{aligned}
\text { deconfined phase: } & \Psi^{\dagger} \Phi \Psi=0, \quad \Phi=\xi\left(\cos \gamma \sigma^{1}+\sin \gamma \sigma^{2}\right)=\xi\left(\begin{array}{cc}
0 & e^{i \gamma} \\
e^{-i \gamma} & 0
\end{array}\right), \\
\text { confined phase: } & \Psi^{\dagger} \Phi \Psi \neq 0, \quad \Phi=\xi \sigma^{3},
\end{aligned}
$$

for eq. (2.25). The unbroken symmetry $\tilde{H}=\mathrm{U}(1)_{Y+W_{3}}$ is further broken to either $\mathbb{Z}_{4}$ or $\mathbb{Z}_{2}$ (unlike the triplet Higgs model beyond the SM in which $\tilde{H}$ should be unbroken). In the deconfined phase, $\Phi$ is invariant under the $\mathbb{Z}_{4}$ symmetry whose minimum element is given by

$$
\left(\mathbb{Z}_{4}\right)_{Y+W_{3}}: \quad\left(e^{i \pi}, e^{i \frac{\pi}{2} \sigma^{3}}\right) \in \mathrm{U}(1)_{Y} \times \mathrm{SU}(2)_{W}: \quad e^{i \pi} e^{i \frac{\pi}{2} \sigma^{3}} \Phi e^{-i \frac{\pi}{2} \sigma^{3}}=\Phi
$$

In the confined phase, $\Phi=\xi \sigma^{3}$ is invariant under the double action of eq. (2.31) generating $\left(\mathbb{Z}_{2}\right)_{Y+W}$ in eq. (2.18). Thus, we have unbroken symmetries

$$
\begin{aligned}
\text { deconfined phase: } & K_{\mathrm{d}}=\left(\mathbb{Z}_{4}\right)_{Y+W_{3}}, \\
\text { confined phase: } & K_{\mathrm{c}}=\left(\mathbb{Z}_{2}\right)_{Y+W},
\end{aligned}
$$

\footnotetext{
${ }^{12}$ In this case, the actual gauge group is $\left[\mathrm{U}(1)_{Y} \times \mathrm{SU}(2)_{W}\right] /\left(\mathbb{Z}_{2}\right)_{Y+W}$, but it is useful to consider the universal covering $\mathrm{U}(1)_{Y} \times \mathrm{SU}(2)_{W}$. The vacuum manifold in eq. (2.28) below is the same. See also the footnote 7 .
} 
consistent with the previous discussion as it should be so. One can easily check that these discrete groups are subgroups of the $\mathrm{U}(1)_{Y+W_{3}}$ group in eq. (2.27). Thus, the vacuum manifolds for the second symmetry breaking are found to be

$$
\begin{aligned}
& \text { deconfined phase: } & \frac{\tilde{H}}{K_{\mathrm{d}}} & =\frac{\mathrm{U}(1)_{Y+W_{3}}}{\left(\mathbb{Z}_{4}\right)_{Y+W_{3}}} \\
& \text { confined phase: } & \frac{\tilde{H}}{K_{\mathrm{c}}} & =\frac{\mathrm{U}(1)_{Y+W_{3}}}{\left(\mathbb{Z}_{2}\right)_{Y+W}},
\end{aligned}
$$

and the lower dimensional homotopy groups are

$$
\begin{array}{rlr}
\text { deconfined: } & \pi_{0}\left(\frac{\tilde{H}}{K_{\mathrm{d}}}\right) \simeq\{0\}, \quad \pi_{1}\left(\frac{\tilde{H}}{K_{\mathrm{d}}}\right) \simeq \mathbb{Z}, \quad \pi_{2}\left(\frac{\tilde{H}}{K_{\mathrm{d}}}\right) \simeq\{0\}, \quad \pi_{3}\left(\frac{\tilde{H}}{K_{\mathrm{d}}}\right) \simeq\{0\}, \\
\text { confined: } & \pi_{0}\left(\frac{\tilde{H}}{K_{\mathrm{c}}}\right) \simeq\{0\}, \quad \pi_{1}\left(\frac{\tilde{H}}{K_{\mathrm{c}}}\right) \simeq \mathbb{Z}, \quad \pi_{2}\left(\frac{\tilde{H}}{K_{\mathrm{c}}}\right) \simeq\{0\}, \quad \pi_{3}\left(\frac{\tilde{H}}{K_{\mathrm{c}}}\right) \simeq\{0\} .
\end{array}
$$

There are only nontrivial first homotopy groups admitting strings, which look the same for the deconfined and confined phases. However, the vacuum manifolds in eq. (2.33) show that the deconfined and confined phases allow 1/4 quantized and 1/2 quantized strings, respectively.

When both the triplet and doublet fields develop VEVs, the unbroken symmetries and physics are the same between the two cases that the triplet (doublet) develops VEVs at high energy and the doublet (triplet) develops VEVs at low energy. In the above discussion, these two cases look different at first glance; for instance, in the former, the doublet field $\Psi$ contains a continuous parameter $\beta$ in eq. (2.16), and in the lattter, the triplet field $\Phi$ contains a continuous parameter $\gamma$ in eq. (2.30). This is just because of a difference of the gauge fixings: for the former (latter) the gauge fixing in eq. (2.6) [(2.25)] was convenient, and these two gauges can be of course transformed to each other by a gauge transformation. In fact, the classification of the deconfined and confined phase is the identical in a gauge invariant description, as can be seen in eq. (2.20),

\section{Strings and monopoles}

In this section, we discuss string and monopole configurations associated with homotopy groups in eq. (2.13) in the absence of the doublet field.

\subsection{Abrikosov-Nielsen-Olesen string and $\mathbb{Z}_{2}$ string}

The ANO string $[63,64]$ is a vortex having a phase winding only in the $\mathrm{U}(1)$ gauge group. In our case, it has the form of

$$
\begin{gathered}
\Phi(r, \theta) \sim \xi\left(\begin{array}{cc}
0 & e^{i \theta} \\
e^{i \theta} & 0
\end{array}\right)=e^{i \theta} \sigma^{1}, \\
A_{i}=0, \quad a_{i} \sim-\frac{1}{e} \frac{\epsilon_{i j} x^{j}}{r^{2}} .
\end{gathered}
$$


This has a unit $\mathrm{U}(1)$ magnetic flux and no $\mathrm{SU}(2)$ flux. This is BPS when we take the BPS limit $\lambda_{e}=e$ and $\lambda_{g}=g$.

Next, we consider a $\mathbb{Z}_{2}$ string which exists in an $\mathrm{SU}(2)$ gauge theory with a complex adjoint field [63]. ${ }^{13}$ It has the form of

$$
\begin{aligned}
\Phi(r, \theta) & \sim \xi\left(\begin{array}{cc}
0 & e^{i \theta} \\
e^{-i \theta} & 0
\end{array}\right)=\xi e^{i \frac{\theta}{2} \sigma^{3}} \sigma^{1} e^{-i \frac{\theta}{2} \sigma^{3}} \\
A_{i} & \sim-\frac{1}{2 g} \frac{\epsilon_{i j} x^{j}}{r^{2}} \sigma^{3}, \quad a_{i}=0
\end{aligned}
$$

The $\mathrm{SU}(2)$ group element $e^{i \frac{\theta}{2} \sigma^{3}}$ varies from 1 to -1 when $\theta$ changes from $\theta=0$ to $\theta=2 \pi$. Thus, the $\mathbb{Z}_{2}$ string connects the center elements \pm 1 of $\mathrm{SU}(2)$ and carries a half amount of the $\mathrm{SU}(2)$ magnetic flux compared with one corresponding to a closed loop in $\mathrm{SU}(2) .{ }^{14}$ This is non-BPS. However, it is stable.

\subsection{Alice strings}

Let us introduce an Alice string discussed in refs. [57-59]. The configuration of an Alice string can be expressed at a large distance as

$$
\begin{aligned}
\Phi(r, \theta) & \sim \xi\left(\begin{array}{cc}
0 & e^{i \theta} \\
1 & 0
\end{array}\right)=\xi e^{i \frac{\theta}{2}}\left(\begin{array}{cc}
0 & e^{+i \frac{\theta}{2}} \\
e^{-i \frac{\theta}{2}} & 0
\end{array}\right)=\xi e^{i \frac{\theta}{2}} e^{+i \frac{\theta}{4} \sigma^{3}} \sigma^{1} e^{-i \frac{\theta}{4} \sigma^{3}}, \\
A_{i} & \sim-\frac{1}{4 g} \frac{\epsilon_{i j} x^{j}}{r^{2}} \sigma^{3}, \quad a_{i} \sim-\frac{1}{2 e} \frac{\epsilon_{i j} x^{j}}{r^{2}}
\end{aligned}
$$

or

$$
\begin{aligned}
\Phi(r, \theta) & \sim \xi\left(\begin{array}{cc}
0 & 1 \\
e^{i \theta} & 0
\end{array}\right)=\xi e^{i \frac{\theta}{2}}\left(\begin{array}{cc}
0 & e^{-i \frac{\theta}{2}} \\
e^{+i \frac{\theta}{2}} & 0
\end{array}\right)=\xi e^{i \frac{\theta}{2}} e^{-i \frac{\theta}{4} \sigma^{3}} \sigma^{1} e^{+i \frac{\theta}{4} \sigma^{3}}, \\
A_{i} & \sim+\frac{1}{4 g} \frac{\epsilon_{i j} x^{j}}{r^{2}} \sigma^{3}, \quad a_{i} \sim-\frac{1}{2 e} \frac{\epsilon_{i j} x^{j}}{r^{2}}
\end{aligned}
$$

with an angular coordinate $\theta$. It carries a half amount of the $\mathrm{U}(1)$ magnetic flux and $1 / 4$ amount of the $\mathrm{SU}(2)$ color magnetic flux, taking a value on $\pm \sigma^{3}$ in this case. It is important to note that the $1 / 4$ quantization of the $\mathrm{SU}(2)$ color magnetic flux of the Alice string is a consequence of $\left(\mathbb{Z}_{4}\right)_{Y+W_{2,3}}$ in eq. (2.12). The Alice string also can be regarded as a half ANO string as well as a half $\mathbb{Z}_{2}$ string.

A full configuration including finite $r$ can be obtained by complementing profile functions in the asymptotic form in eq. (3.3). See appendix B.

\footnotetext{
${ }^{13}$ In the original case [63], $\mathrm{U}(1)$ is a global symmetry.

${ }^{14}$ Since the center does not act on the triplet in the adjoint action, the actual gauge group is $\mathrm{SO}(3)=$ $\mathrm{SU}(2) / \mathbb{Z}_{2}$ unless we introduce a doublet field. In this case, eq. (3.2) gives a closed loop in $\mathrm{SO}(3)$. While it carries a half amount of flux in terms of $\mathrm{SU}(2)$, it carries a unit flux in terms of $\mathrm{SO}(3)$ because it is given by a closed loop in $\mathrm{SO}(3)$. In this paper, however, we also consider the doublet, and so our gauge group is $\mathrm{SU}(2)$ rather than $\mathrm{SO}(3)$.
} 
These two configurations are merely typical configurations belonging to a continuous family of more general configurations, given by

$$
\begin{aligned}
\Phi(r, \theta) & \sim \xi U\left(\begin{array}{cc}
0 & e^{i \theta} \\
1 & 0
\end{array}\right) U^{\dagger}=\xi e^{i \frac{\theta}{2}} e^{+i \frac{\theta}{4} \hat{\sigma}} \sigma^{1} e^{-i \frac{\theta}{4} \hat{\sigma}}, \\
A_{i} & \sim-\frac{1}{4 g} \frac{\epsilon_{i j} x^{j}}{r^{2}} \hat{\sigma}, \quad a_{i} \sim-\frac{1}{2 e} \frac{\epsilon_{i j} x^{j}}{r^{2}},
\end{aligned}
$$

where $U$ is defined in eq. (2.17) and $\hat{\sigma}$ is defined by

$$
\hat{\sigma}=U \sigma^{3} U^{\dagger}=\sin \beta \sigma^{2}+\cos \beta \sigma^{3} .
$$

Here, $\beta$ is a $\mathrm{U}(1)$ modulus characterizing an $\mathrm{SU}(2)$ color magnetic flux between $\sigma^{2}$ and $\sigma^{3}$, and $\beta=0(\beta=\pi)$ corresponds to the configuration in eq. (3.3) [eq. (3.4)].

On the other hand, anti-strings carrying a $-1 / 2 \mathrm{U}(1)$ magnetic flux and $1 / 4 \mathrm{SU}(2)$ magnetic flux can be constructed as

$$
\begin{aligned}
\Phi(r, \theta) & \sim \xi\left(\begin{array}{cc}
0 & 1 \\
e^{-i \theta} & 0
\end{array}\right)=\xi e^{-i \frac{\theta}{2}}\left(\begin{array}{cc}
0 & e^{+i \frac{\theta}{2}} \\
e^{-i \frac{\theta}{2}} & 0
\end{array}\right)=\xi e^{-i \frac{\theta}{2}} e^{+i \frac{\theta}{4} \sigma^{3}} \sigma^{1} e^{-i \frac{\theta}{4} \sigma^{3}} \\
A_{i} & \sim-\frac{1}{4 g} \frac{\epsilon_{i j} x^{j}}{r^{2}} \sigma^{3}, \quad a_{i} \sim+\frac{1}{2 e} \frac{\epsilon_{i j} x^{j}}{r^{2}}
\end{aligned}
$$

or

$$
\begin{aligned}
\Phi(r, \theta) & \sim \xi\left(\begin{array}{cc}
0 & e^{-i \theta} \\
1 & 0
\end{array}\right)=\xi e^{-i \frac{\theta}{2}}\left(\begin{array}{cc}
0 & e^{-i \frac{\theta}{2}} \\
e^{+i \frac{\theta}{2}} & 0
\end{array}\right)=\xi e^{-i \frac{\theta}{2}} e^{-i \frac{\theta}{4} \sigma^{3}} \sigma^{1} e^{+i \frac{\theta}{4} \sigma^{3}} \\
A_{i} & \sim+\frac{1}{4 g} \frac{\epsilon_{i j} x^{j}}{r^{2}} \sigma^{3}, \quad a_{i} \sim+\frac{1}{2 e} \frac{\epsilon_{i j} x^{j}}{r^{2}}
\end{aligned}
$$

Again, more generally, they belong to a continuous family of configuration:

$$
\begin{aligned}
\Phi(r, \theta) & \sim \xi U\left(\begin{array}{cc}
0 & 1 \\
e^{-i \theta} & 0
\end{array}\right) U^{\dagger}=\xi e^{-i \frac{\theta}{2}} e^{+i \frac{\theta}{4} \hat{\sigma}} \sigma^{1} e^{-i \frac{\theta}{4} \hat{\sigma}}, \\
A_{i} & \sim-\frac{1}{4 g} \frac{\epsilon_{i j} x^{j}}{r^{2}} \hat{\sigma}, \quad a_{i} \sim+\frac{1}{2 e} \frac{\epsilon_{i j} x^{j}}{r^{2}}
\end{aligned}
$$

with $U$ defined in eq. (2.17) and $\hat{\sigma}$ defined in eq. (3.6).

Let us briefly show the Alice property. The triplet field approaches $\Phi(r \rightarrow \infty, \theta=$ $0)=\xi \sigma^{1}$ asymptotically along the $x^{1}$-axis $(\theta=0)$, and the $\theta$-dependence is given by

$$
\begin{aligned}
\Phi(r \rightarrow \infty, \theta) & \sim e^{i e \int \mathbf{a} \cdot \mathbf{d} \mathbf{l}} P e^{i g \int \mathbf{A} \cdot \mathbf{d l}} \Phi(r \rightarrow \infty, 0) P e^{-i g \int \mathbf{A} \cdot \mathbf{d} \mathbf{l}} \\
& \sim U_{0}(\theta) U_{3}(\theta) \Phi(r \rightarrow \infty, 0) U_{3}^{-1}(\theta),
\end{aligned}
$$

where the holonomies are defined as

$$
U_{0}(\theta)=e^{i e \int_{0}^{\theta} \mathbf{a} \cdot \mathbf{d} \mathbf{l}}=e^{i \frac{\theta}{2}} \in \mathrm{U}(1), \quad U_{3}(\theta)=P e^{i g \int_{0}^{\theta} \mathbf{A} \cdot \mathbf{d} \mathbf{l}}=e^{i \frac{\theta}{4} \sigma^{3}} \in \mathrm{SU}(2) .
$$


These can be understood by using the condition of topological vortex where the order parameter $\Phi$ is covariantly constant at large distances $\left(\mathcal{D}_{i} \Phi \rightarrow 0\right.$ as $\left.r \rightarrow \infty\right)$. When we encircle the string by an angle $\theta$, the unbroken $\mathrm{U}(1)$ generator $Q$ transforms as

$$
Q_{\theta}=U_{3}(\theta) Q_{0} U_{3}(\theta)^{-1}
$$

with $Q_{0}=\sigma^{1}$. We thus have a sign flip of the $\mathrm{U}(1)$ generator after one complete encirclement as

$$
Q_{2 \pi}=-Q_{0}
$$

implying that the unbroken generator is not singly defined around the string, which is nothing but the Alice property and sometimes called a topological obstruction.

For later convenience, let us give a doubly-wound Alice string configuration, which is the most important among multiple Alice string configurations. It has the form of

$$
\begin{aligned}
\Phi(r, \theta) & \sim \xi\left(\begin{array}{cc}
0 & e^{2 i \theta} \\
1 & 0
\end{array}\right)=\xi e^{i \theta}\left(\begin{array}{cc}
0 & e^{+i \theta} \\
e^{-i \theta} & 0
\end{array}\right)=\xi e^{i \theta} e^{i \frac{\theta}{2} \sigma^{3}} \sigma^{1} e^{-i \frac{\theta}{2} \sigma^{3}}, \\
A_{i} & \sim-\frac{1}{2 g} \frac{\epsilon_{i j} x^{j}}{r^{2}} \sigma^{3}, \quad a_{i} \sim-\frac{1}{e} \frac{\epsilon_{i j} x^{j}}{r^{2}}
\end{aligned}
$$

or

$$
\begin{aligned}
\Phi(r, \theta) & \sim \xi\left(\begin{array}{cc}
0 & 1 \\
e^{2 i \theta} & 0
\end{array}\right)=\xi e^{i \theta}\left(\begin{array}{cc}
0 & e^{-i \theta} \\
e^{+i \theta} & 0
\end{array}\right)=\xi e^{i \theta} e^{i \frac{\theta}{2} \sigma^{3}} \sigma^{1} e^{-i \frac{\theta}{2} \sigma^{3}}, \\
A_{i} & \sim+\frac{1}{2 g} \frac{\epsilon_{i j} x^{j}}{r^{2}} \sigma^{3}, \quad a_{i} \sim-\frac{1}{e} \frac{\epsilon_{i j} x^{j}}{r^{2}}
\end{aligned}
$$

for a specific orientation and

$$
\begin{aligned}
\Phi(r, \theta) & \sim \xi U\left(\begin{array}{cc}
0 & e^{2 i \theta} \\
1 & 0
\end{array}\right) U^{\dagger}=\xi e^{i \theta} e^{+i \frac{\theta}{2} \hat{\sigma}} \sigma^{1} e^{-i \frac{\theta}{2} \hat{\sigma}} \\
A_{i} & \sim-\frac{1}{2 g} \frac{\epsilon_{i j} x^{j}}{r^{2}} \hat{\sigma}, \quad a_{i} \sim-\frac{1}{e} \frac{\epsilon_{i j} x^{j}}{r^{2}}
\end{aligned}
$$

with $U$ defined in eq. (2.17) and $\hat{\sigma}$ defined in eq. (3.6), for more general configuration. Here, $\beta$ is again a $\mathrm{U}(1)$ modulus characterizing an $\mathrm{SU}(2)$ color magnetic flux between $\sigma^{2}$ and $\sigma^{3}$. Each carries a unit $\mathrm{U}(1)$ magnetic flux and a half $\mathrm{SU}(2)$ magnetic flux, which are just twice of a single Alice string. This can also be regarded as a composite of one ANO vortex and one $\mathbb{Z}_{2}$ vortex. This should be BPS, since it is just twice of one Alice string (although a $\mathbb{Z}_{2}$ string is not BPS). The unbroken generator $Q$ is singly defined around the doubly-wound Alice string, unlike a single Alice string.

\subsection{Alice monopoles}

An Alice string carries a U(1) modulus $\beta$ in eq. (3.5), corresponding to the internal direction of the flux [58]. Let us consider a closed Alice string as in figure 2 (a). The black arrows 


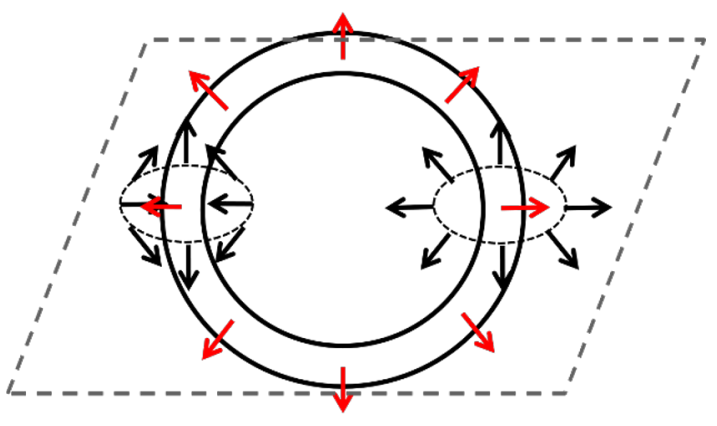

(a)

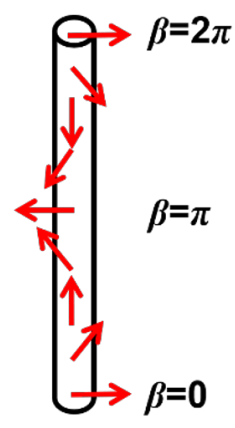

(b)

Figure 2. A monopoles as a twisted Alice string. (a) an isolated monopole as a twisted Alice ring along which the $\mathrm{U}(1)$ modulus is twisted once. (b) a monopole on a straight Alice string along which the $\mathrm{U}(1)$ modulus is twisted once.

represent the phase winding of the string. The antipodal points of the ring (cut by a plane in the figure) are a pair of a vortex and anti-vortex, and therefore the ring is usually unstable to shrink. When a $\mathrm{U}(1)$ modulus $\beta$ (represented by red arrows) is twisted along the closed Alice string as in figure 2 (a), it can be stable depending on a parameter region and is called a vorton, as discussed below. This twisted Alice ring carries a magnetic monopole charge.

In ref. [52], Ruostekoski and Anglin found such a stable magnetic monopole solution in the theory without gauge fields (in the context of spinor Bose-Einstein condensates), which is therefore a global monopole.

A similar case for local monopoles were studied in the $\mathrm{SO}(3)$ gauge theory with a fiveplet real scalar field (real symmetric traceless tensor) [41, 42, 77] admitting a conventional Alice string, in which the vacuum manifold is $\mathbb{R} P^{2} \simeq S^{2} / \mathbb{Z}_{2}$ rather than eq. (2.12). In this case, it was observed that a usual spherical 't Hooft-Polyakov monopole decays into a twisted Alice ring (in a certain parameter region).

Let us discuss the issue of the stability. On the plane cutting the ring, the vortex and anti-vortex are given by eqs. (3.3) and (3.7), respectively. Note that because of the U(1) modulus twist, the anti-vortex is not the one in eq. (3.8). The $\mathrm{SU}(2)$ fluxes are the same, while the $\mathrm{U}(1)$ fluxes are opposite to each other. In general, the $\mathrm{U}(1)$ gauge field mediates an attraction between a vortex and anti-vortex, while the $\mathrm{SU}(2)$ gauge fields mediates a repulsion between the same magnetic fluxes. Thus, at least when the $\mathrm{SU}(2)$ gauge field is lighter than the $\mathrm{U}(1)$ gauge field $(g<e)$ and in the type-II regime (implying that Higgs fields are heavier than gauge fields), they can repel and contribute to the stability.

By cutting the Alice ring and stretch it to a straight string (along the $z$-direction), we obtain an Alice string along which the U(1) modulus is twisted once, as in figure 2 (b):

$$
\begin{aligned}
\Phi(r, \theta) & \sim \xi e^{i \frac{\theta}{2}} e^{+i \frac{\theta}{4} \hat{\sigma}} \sigma^{1} e^{-i \frac{\theta}{4} \hat{\sigma}} \\
A_{i} & \sim-\frac{1}{4 g} \frac{\epsilon_{i j} x^{j}}{r^{2}} \hat{\sigma}, \quad a_{i} \sim-\frac{1}{2 e} \frac{\epsilon_{i j} x^{j}}{r^{2}} \\
\hat{\sigma} & =\sin \beta(z) \sigma^{2}+\cos \beta(z) \sigma^{3} .
\end{aligned}
$$


In this case, the monopole charge density is broadly present where the $\mathrm{U}(1)$ modulus is twisted. Namely, the $\mathrm{U}(1)$ modulus $\beta$ of the string winds once along it from $\beta=0$ at $z \rightarrow-\infty$ to $\beta=0$ at $z \rightarrow+\infty$. However, this charge cannot be localized and is spread over the string in order to reduce the gradient energy. ${ }^{15}$

\section{Confinement of Alice strings and monopoles}

In this section, we first discuss the $\mathrm{AB}$ phase around an Alice string. We then introduce $\mathrm{AB}$ defects appearing when the doublet field develops VEVs, and discuss the bulk-soliton moduli locking in the deconfined phase. After classifying configurations of two strings by $\mathrm{AB}$ phases of the doublet field, the confinement of Alice strings and decays of an ANO string and $\mathbb{Z}_{2}$ string are discussed in the confined phase. Finally, we discuss the confinement of monopoles.

\subsection{Aharonov-Bohm phases around an Alice string}

When the doublet field $\Psi=(a, b)^{T}$ encircles around an Alice string, it receives an $\mathrm{AB}$ phase as

$$
\Psi_{\theta}=e^{i \frac{e}{2} \int_{0}^{\theta} a \cdot d l}\left(P e^{i g \int_{0}^{\theta} A \cdot d l}\right)\left(\begin{array}{l}
a \\
b
\end{array}\right) .
$$

This phase depends on the $\mathrm{U}(1)$ modulus of the Alice string. For instance, it is given by

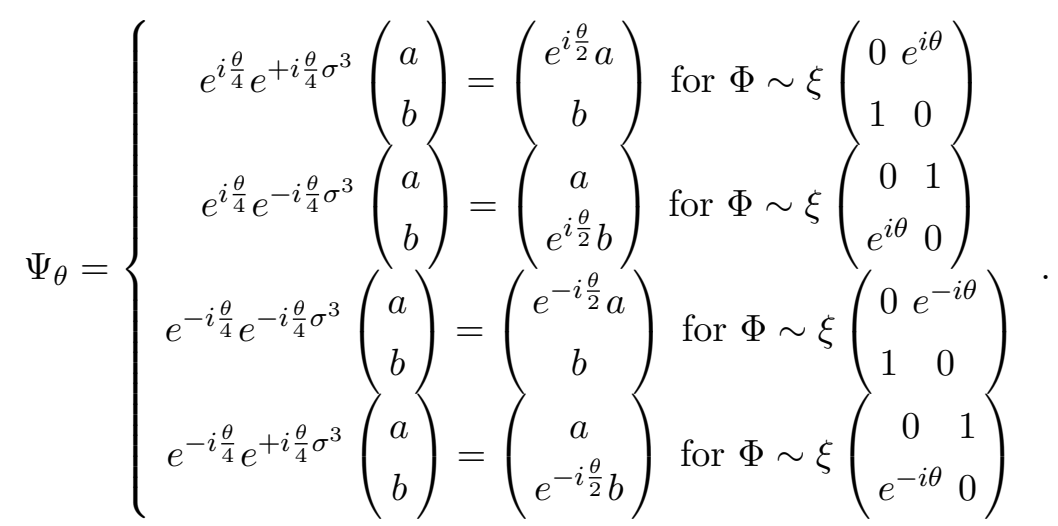

As it can be noticed that after a complete encirclement, the doublet field gets an AB phase as

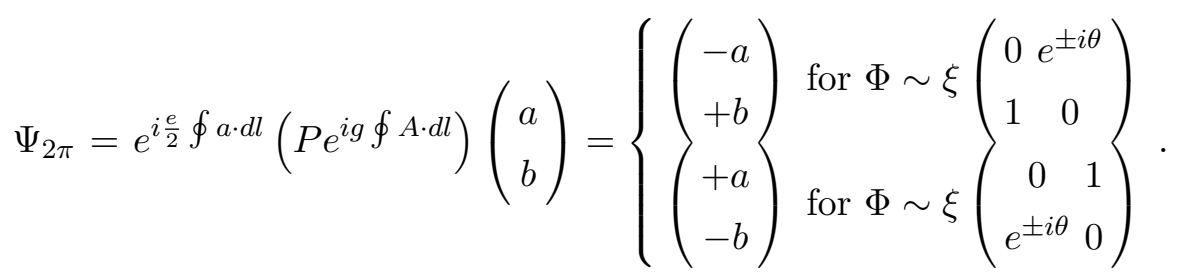

The important observation is that the doublet field can feel the flux (modulus) of the Alice string from infinitely far away from it by encircling around it. From this fact, we say that color is not confined for a single Alice string.

\footnotetext{
${ }^{15}$ If one direction of the space is compactified on $S^{1}$, and the string winds around it, the configuration is stable.
} 


\section{$4.2 \quad$ Bulk-soliton moduli locking}

When the doublet field $\Psi$ develops VEVs in the absence of an Alice string, the VEV can be arbitrary:

$$
\Psi=\left(\begin{array}{l}
a \\
b
\end{array}\right), \quad|a|^{2}+|b|^{2}=v^{2},
$$

parametrizing $S^{3}$ (if we turn off the interaction between the triplet and doublet fields).

On the other hand, in the presence of a single Alice string, the VEVs of $\Psi$ is singlevalued for

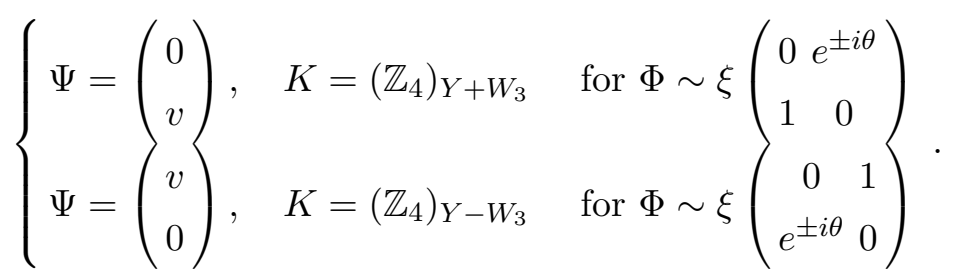

More generally, the single-valuedness condition is given by

$$
\Psi=U\left(\begin{array}{l}
0 \\
v
\end{array}\right)=\left(\begin{array}{c}
i v \sin \frac{\beta}{2} \\
v \cos \frac{\beta}{2}
\end{array}\right), \quad K=\left(\mathbb{Z}_{4}\right)_{Y+W_{2,3}} \quad \text { for } \Phi \sim \xi U\left(\begin{array}{cc}
0 & e^{ \pm i \theta} \\
1 & 0
\end{array}\right) U^{\dagger}
$$

with $U$ defined in eq. (2.17), for the (anti-)Alice string configurations in eq. (3.5) [(3.9)]. Here, $\beta=0$ and $\beta=\pi$ correspond to the first and second lines in eq. (4.5), respectively (up to the constant factor $i$ ). Otherwise, the doublet field cannot be single-valued. Thus, we have found that the U(1) modulus of the Alice string and the vacuum moduli of the doublet fields are locked. We call this the bulk-soliton moduli locking mechanism. We should point out that $\Psi$ is in the deconfined phase satisfies $\Psi^{\dagger} \Phi \Psi=0$ as can be seen in eq. (2.20).

We have two schemes to interpret this phenomenon. When the triplet VEVs are much larger than the doublet VEVs, the symmetry breaking by the triplet field occurs at high energy and subsequently the symmetry breaking by the doublet field occurs at low energy. This is the scheme that we are mainly considering in this paper. In this case, we can fix a triplet configuration (with a string) as a background. Then, if we turn on the doublet VEVs gradually, they develop as in eq. (4.5) or more generally as in eq. (4.6). Thus, the doublet choses the deconfined phase. It is interesting that the phase of matter is determined by the presence of a soliton.

In the opposite scheme discussed in section 2.4, in which the doublet VEVs are much larger than the triplet VEVs, the symmetry breaking by the doublet field occurs at high energy and subsequently the symmetry breaking by the triplet field occurs at low energy. In this case, if one creates an Alice string, its $\mathrm{U}(1)$ modulus $\beta$ should be aligned to the doublet field, as eq. (4.5) or more generally as eq. (4.6). Namely, the flux is determined by the doublet moduli in the bulk. This can be understood from the vacuum manifold in the first line in eq. (2.33) allowing strings. In this case, one can observe that strings are purely ANO-like, in contrast to eq. (2.12) in which the $\mathrm{U}(1)$ group is present in the denominator of the coset space, endowing the $\mathrm{U}(1)$ modulus to the string. When one creates multiple Alice strings, all of their U(1) moduli must be aligned to the doublet field. In other words, the $\mathrm{U}(1)$ moduli of strings are killed by the doublet field. 


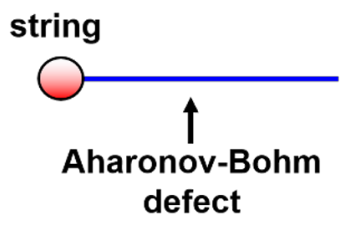

(a)

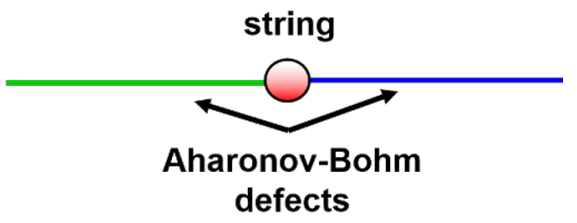

(b)

Figure 3. Aharonov-Bohm defects attached to a string. (a) one or (b) two AB defect(s) attached to cancel the nontrivial $\mathrm{AB}$ phase(s) of the doublet fields around the string. (a) is the case of an Alice string. (b) corresponds to the case of an ANO string or $\mathbb{Z}_{2}$ string. The two AB defects are generated in the two components of the doublet field, and in general they have different tension depending on the VEV of each component.

\subsection{Aharonov-Bohm defects}

Next, let us consider the confined phase. ${ }^{16}$ Then, it is inevitable for the doublet fields to receive an $\mathrm{AB}$ phase which must be canceled for the single-valuedness as

$$
\Psi \sim\left\{\left\{\begin{array}{c}
a e^{ \pm i \frac{\theta}{2}} f( \pm(\theta-\alpha)) \\
b \\
a \\
b e^{ \pm i \frac{\theta}{2}} f( \pm(\theta-\alpha))
\end{array}\right) \text { for } \Phi \sim \xi\left(\begin{array}{cc}
0 & e^{ \pm i \theta} \\
1 & 0
\end{array}\right)\right.
$$

where the factors $e^{ \pm i \frac{\theta}{2}}$ come from the AB-phase, and $\alpha$ corresponds to the direction of the $\mathrm{AB}$ defect. Here, for the single-valuedness of $\Psi$, we have introduced a multi-valued function $f(\theta)$ on $S^{1}$ which changes the sign under a $2 \pi$ shift: $f(\theta+2 \pi)=-f(\theta)$. This creates a soliton attached to the Alice string as schematically shown in figure $3(\mathrm{a})$. The existence of the soliton is topologically supported by the nontrivial zeroth homotopy group in eq. (2.22) in the confined phase. We called such a soliton as an AB defect [59].

There are two ways to create an $\mathrm{AB}$ defect.

1. An ansatz for the doublet for the first case is to take $f(\theta)=e^{i \phi(\theta) / 2}[59]$ :

$$
\Psi_{\mathrm{DW}} \sim \xi_{\psi}\left(\begin{array}{c}
e^{i\left(\frac{\theta+\phi(\theta)}{2}\right)} \\
0
\end{array}\right), \phi(0)=0, \phi(2 \pi)=-2 \pi,
$$

where $\phi(\theta)$ is a decreasing function and the boundary condition keeps the doublet single-valued. We substitute this ansatz to the Hamiltonian density in the presence of the Alice string configuration in eq. (3.3) at large distances, where the potentials are given in eqs. (2.4) and (2.5). We thus obtain the effective Hamiltonian of the doublet

$$
\mathcal{H}_{\mathrm{DW}} / \xi_{\psi}^{2} \sim \frac{1}{4}\left[\left(\partial_{i} \phi\right)^{2}+8 \mu_{\psi}^{2}(1-\cos \phi)\right], \quad \mu_{\psi}^{2}=\xi \mu,
$$

which is nothing but the sine-Gordon model.

\footnotetext{
${ }^{16} \mathrm{Or}$, we enforce the doublet to have a VEV on the opposite side even in the deconfined phase.
} 
2. The second case is to consider a kink configuration by a real function $f$ varying from +1 to -1 at $\theta=0$ to $\theta=2 \pi$.

In either case, the $\mathrm{AB}$ defect is a linearly extended to infinity, with having infinite energy in infinite space. So it should decay and the configurations in eq. (4.7) go to those in eq. (4.5) by changing the orientation of $\Psi$ in the internal space, and the phase is in the deconfined phase. We conclude that the bulk-soliton moduli locking mechanism is energetically realized.

On the other hand, there appear two $\mathrm{AB}$ defects around an ANO string and a $\mathbb{Z}_{2}$ string as in figure $3(\mathrm{~b})$, since the both components of the doublet field $\Psi$ receive AB phases around those strings. The doublet fields behave around an ANO string as

$$
\Psi \sim\left(\begin{array}{c}
a e^{i \frac{\theta}{2}} f_{1}(\theta) \\
b e^{i \frac{\theta}{2}} f_{2}(\theta-\alpha)
\end{array}\right) \text { for } \Phi(r, \theta) \sim \xi\left(\begin{array}{cc}
0 & e^{i \theta} \\
e^{i \theta} & 0
\end{array}\right)
$$

where $f_{1}$ and $f_{2}$ are multi-valued functions, $\alpha$ controls the relative directions of the $\mathrm{AB}$ defect (we have taken a constant in the upper component to be zero without loss of generality). Energetically, it may be natural to consider $\alpha=\pi$ as in figure 3(b). The two different $\mathrm{AB}$ defects are present in the two different components of the doublet field $\Psi$, and so they have different tensions in general, depending on $a$ and $b$.

Similarly, the doublet fields behave around a $\mathbb{Z}_{2}$ string as

$$
\Psi \sim\left(\begin{array}{c}
a e^{i \frac{\theta}{2}} f_{1}(\theta) \\
b e^{-i \frac{\theta}{2}} f_{2}(-\theta+\alpha)
\end{array}\right) \text { for } \Phi(r, \theta) \sim \xi\left(\begin{array}{cc}
0 & e^{i \theta} \\
e^{-i \theta} & 0
\end{array}\right)
$$

with multi-valued functions $f_{1}$ and $f_{2}$.

In these cases, it is impossible to eliminate the two $\mathrm{AB}$ defects simultaneously in any choice of the doublet VEVs, although one can eliminate at least one AB defect by taking either $a=0$ or $b=0$.

In the case of $q=0$, one cannot eliminate an $\mathrm{AB}$ defect.

\subsection{Classification of configurations of two strings}

When the double VEVs are much larger than the triplet VEVs, we can fix a doublet configuration as a background. Let us consider the confined phase in which the both components of the doublet are nonzero and the $\mathbb{Z}_{4}$ symmetry is broken to $\mathbb{Z}_{2}$. In this case, Alice strings are inevitably attached by $\mathrm{AB}$ defects. Here we discuss that configurations of two strings can be classified by the $\mathrm{AB}$ phases of the doublet field encircling around the two strings, as in figure 4.

The configurations of two strings can be classified into either the confinement [figure 4(a)] or the decay [figure 4(b)], depending on $\mathrm{AB}$ phases of the doublet field encircling around the strings. Without loss of generality, let us consider the case that when the doublet field $\Psi=(a, b)^{T}$ encircles the right string along a smaller loop, it becomes $\Psi=(-a, b)^{T}$ which is not single-valued if there is no $\mathrm{AB}$ defect, implying the existence of an $\mathrm{AB}$ defect attached to it. Next, when the doublet field $\Psi=(a, b)^{T}$ encircles the left string, let us 


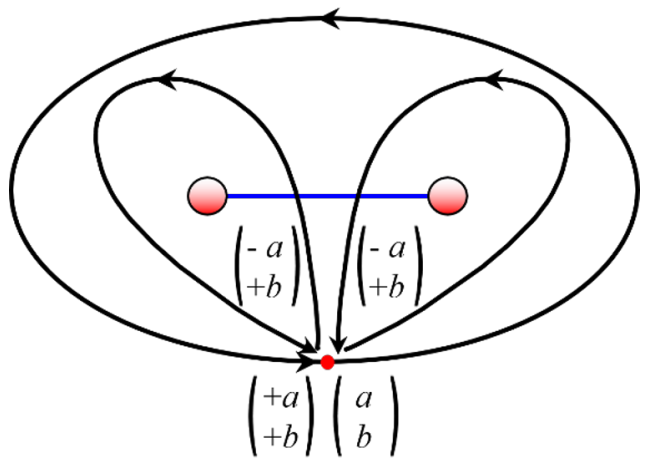

(a)

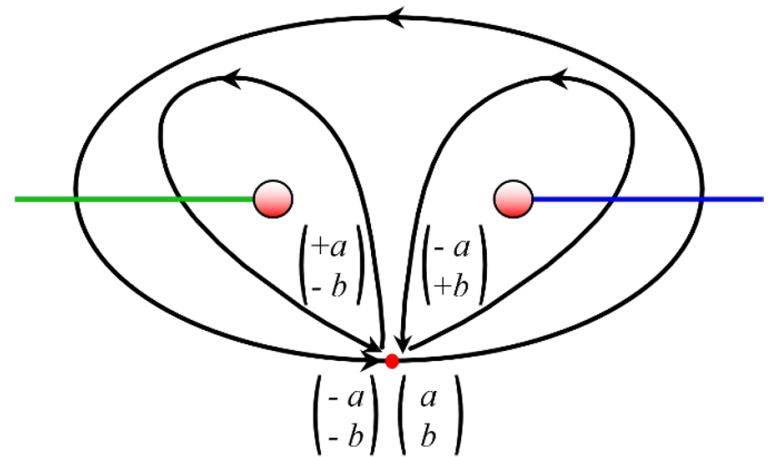

(b)

Figure 4. Configurations of two strings classified by $\mathrm{AB}$ phases around the strings. Configurations of two strings can be classified into either (a) the confinement or (b) the decay, depending on $\mathrm{AB}$ phases of the doublet field encircling around the strings. (a) When the doublet field $\Psi=(a, b)^{T}$ encircles the right (left) string along a smaller loop, it becomes $\Psi=(-a, b)^{T}$ which is not singlevalued if there is no $\mathrm{AB}$ defect, implying the existence of an $\mathrm{AB}$ defect attached to each of them. When the doublet field $\Psi=(a, b)^{T}$ encircles the both strings together (along the large loop), it comes back to $\Psi=(a, b)^{T}$ which is single-valued, implying the absence of $\mathrm{AB}$ defect crossing the large loop. Therefore, one $\mathrm{AB}$ defect connects the two strings, corresponding to the confinement. (b) When the doublet field $\Psi=(a, b)^{T}$ encircles the right (left) string, it becomes $\Psi=(-a, b)^{T}$ $\left(\Psi=(a,-b)^{T}\right)$ which is not single-valued if there is no AB defect, implying the existence of a different $\mathrm{AB}$ defect attached to each of them. When the doublet field $\Psi=(a, b)^{T}$ encircles the both strings together (along the large loop), it becomes $\Psi=(-a,-b)^{T}$ which is not yet single-valued, implying the existence of the two kinds of $\mathrm{AB}$ defects crossing the large loop. No $\mathrm{AB}$ defect connects the two strings, corresponding to the decay.

suppose that it becomes either (a) $\Psi=(-a, b)^{T}$ or $(\mathrm{b}) \Psi=(a,-b)^{T}$. In either case, an $\mathrm{AB}$ defect of a different type is attached to the left string.

In the case of (a), the two $\mathrm{AB}$ defects attached to the two strings are of the same type, and so they can be connected. In fact, if the doublet field $\Psi=(a, b)^{T}$ encircles the both strings together along the larger loop in figure 4 (a), it comes back to $\Psi=(a, b)^{T}$ which is single-valued. This shows the absence of AB defect crossing the large loop, implying that the two strings must be connected by a single AB defect. This case implies the confinement of the two strings.

On the other hand, in the case of (b), the two $\mathrm{AB}$ defects attached to the two strings are of the different type, and so they cannot be connected. In fact, if the doublet field $\Psi=(a, b)^{T}$ encircles the both strings together along the larger loop in figure 4(b), it becomes $\Psi=(-a,-b)^{T}$ which is not yet single-valued, implying the existence of the two kinds of $\mathrm{AB}$ defects crossing the large loop. Thus, no $\mathrm{AB}$ defect connects the two strings, implying that the two strings are pulled to the opposite (or different) directions.

In the following subsections, we discuss the confinement in figure 4(a) and the decay in figure 4(b), respectively. 


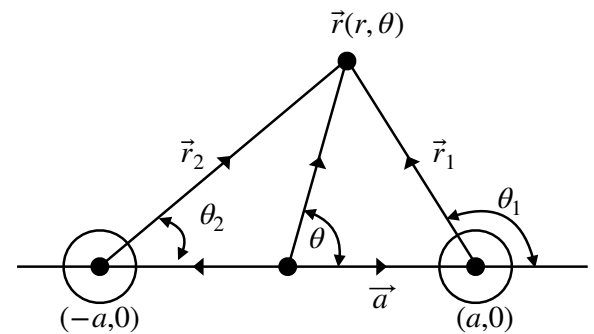

Figure 5. Configuration of two strings. The right (left) strings are placed at $(a, 0)[(-a, 0)]$. $(r, \theta)$ are polar coordinates from the origin, and $\left(r_{1}, \theta_{1}\right)\left[\left(r_{2}, \theta_{2}\right)\right]$ are polar coordinates from $(a, 0)$ $[(-a, 0)]$.

\subsection{Confinement of Alice strings}

First, let us discuss the case of confinement corresponding to figure 4(a). There are two types of the confinement: 1) a pair of an Alice string and anti-Alice string (colorless confinement) and 2) a pair of the same type of Alice strings (colorful confinement). Let us discuss them one by one.

1) A pair of an Alice string and anti-Alice string (colorless confinement). Let us consider a pair of configurations

$$
\begin{aligned}
\Phi_{1}(r, \theta) & \sim \xi\left(\begin{array}{cc}
0 & e^{i \theta_{1}} \\
1 & 0
\end{array}\right)=\xi e^{i \frac{\theta_{1}}{2}} e^{+i \frac{\theta_{1}}{4} \sigma^{3}} \sigma^{1} e^{-i \frac{\theta_{1}}{4} \sigma^{3}}, \\
A_{1, i} & \sim-\frac{1}{4 g} \frac{\epsilon_{i j} x_{1}^{j}}{r_{1}^{2}} \sigma^{3}, \quad a_{1, i} \sim-\frac{1}{2 e} \frac{\epsilon_{i j} x_{1}^{j}}{r_{1}^{2}}, \\
\Psi_{1} & \sim\left(\begin{array}{c}
a e^{i \frac{\theta_{1}}{2}} f_{1}\left(\theta_{1}-\alpha_{1}\right) \\
b
\end{array}\right)
\end{aligned}
$$

and

$$
\begin{aligned}
\Phi_{2}(r, \theta) & \sim \xi\left(\begin{array}{cc}
0 & e^{-i \theta_{2}} \\
1 & 0
\end{array}\right)=\xi e^{-i \frac{\theta_{2}}{2}} e^{-i \frac{\theta_{2}}{4} \sigma^{3}} \sigma^{1} e^{+i \frac{\theta_{2}}{4} \sigma^{3}} \\
A_{2, i} & \sim+\frac{1}{4 g} \frac{\epsilon_{i j} x_{2}^{j}}{r_{2}^{2}} \sigma^{3}, \quad a_{2, i} \sim+\frac{1}{2 e} \frac{\epsilon_{i j} x_{2}^{j}}{r_{2}^{2}} \\
\Psi_{2} & \sim\left(\begin{array}{c}
a e^{-i \frac{\theta_{2}}{2}} f_{1}\left(\theta_{2}-\alpha_{2}\right) \\
b
\end{array}\right)
\end{aligned}
$$

with constants $\alpha_{1}$ and $\alpha_{2}$ describing the directions of the AB defects. Here $(r, \theta)$ are polar coordinates from the origin, and $\left(r_{1}, \theta_{1}\right)\left[\left(r_{2}, \theta_{2}\right)\right]$ are polar coordinates from $(a, 0)$ [ $\left.(-a, 0)\right]$. The configurations $\left(\Phi_{1}, A_{1}, a_{1}, \Psi_{1}\right)$ and $\left(\Phi_{2}, A_{2}, a_{2}, \Psi_{2}\right)$ denote a vortex placed at $(a, 0)$ and anti-vortex at $(-a, 0)$. These two vortices are connected by an AB defect as in figure 4(a), 
if one considers the total configuration constructed by an Abrikosov-like ansatz,

$$
\begin{aligned}
& \Phi_{\mathrm{tot}} \sim \xi\left(\begin{array}{cc}
0 & e^{i\left(\theta_{1}-\theta_{2}\right)} \\
1 & 0
\end{array}\right) \rightarrow \xi\left(\begin{array}{ll}
0 & 1 \\
1 & 0
\end{array}\right), \\
& A_{\mathrm{tot}}=A_{1}+A_{2} \rightarrow 0, \quad a_{\mathrm{tot}}=a_{1}+a_{2} \rightarrow 0, \\
& \Psi_{\mathrm{tot}} \sim\left(\begin{array}{c}
a e^{i \frac{\theta_{1}-\theta_{2}}{2}} f_{1}\left(\theta_{1}-\theta_{2}-\pi\right) \\
b
\end{array}\right) \rightarrow\left(\begin{array}{l}
a \\
b
\end{array}\right) .
\end{aligned}
$$

This configuration corresponds to take $\alpha_{1}=\pi, \alpha_{2}=0$ in the individual configurations in order to connect them. In this case, they annihilate in pair as indicated by arrows in the above equation, and the final state is the vacuum $\Phi=\sigma^{1}$ and $\Psi=$ constant as expected.

2) A pair of the same type of Alice strings (colorful confinement).

Next, let us consider a pair of configurations

$$
\begin{aligned}
\Phi_{1}(r, \theta) & \sim \xi\left(\begin{array}{cc}
0 & e^{i \theta_{1}} \\
1 & 0
\end{array}\right)=\xi e^{i \frac{\theta_{1}}{2}} e^{+i \frac{\theta_{1}}{4} \sigma^{3}} \sigma^{1} e^{-i \frac{\theta_{1}}{4} \sigma^{3}}, \\
A_{1, i} & \sim-\frac{1}{4 g} \frac{\epsilon_{i j} x_{1}^{j}}{r_{1}^{2}} \sigma^{3}, \quad a_{1, i} \sim-\frac{1}{2 e} \frac{\epsilon_{i j} x_{1}^{j}}{r_{1}^{2}}, \\
\Psi_{1} & \sim\left(\begin{array}{c}
a e^{i \frac{\theta_{1}}{2}} f_{1}\left(\theta_{1}-\alpha_{1}\right) \\
b
\end{array}\right)
\end{aligned}
$$

and

$$
\begin{aligned}
\Phi_{2}(r, \theta) & \sim \xi\left(\begin{array}{cc}
0 & e^{i \theta_{2}} \\
1 & 0
\end{array}\right)=\xi e^{i \frac{\theta_{2}}{2}} e^{+i \frac{\theta_{2}}{4} \sigma^{3}} \sigma^{1} e^{-i \frac{\theta_{2}}{4} \sigma^{3}}, \\
A_{2, i} & \sim-\frac{1}{4 g} \frac{\epsilon_{i j} x_{2}^{j}}{r_{2}^{2}} \sigma^{3}, \quad a_{2, i} \sim-\frac{1}{2 e} \frac{\epsilon_{i j} x_{2}^{j}}{r_{2}^{2}} \\
\Psi_{2} & \sim\left(\begin{array}{c}
a e^{i \frac{\theta_{2}}{2}} f_{1}\left(\theta_{2}-\alpha_{2}\right) \\
b
\end{array}\right) .
\end{aligned}
$$

The coordinates are the same with figure 5 , and the configurations $\left(\Phi_{1}, A_{1}, a_{1}\right)$ and $\left(\Phi_{2}, A_{2}, a_{2}\right)$ denote vortices of the same kind placed at $(a, 0)$ and $(-a, 0)$. Again, these two vortices are also connected by an $\mathrm{AB}$ defect as in figure 4(a), if the total configuration is constructed by an Abrikosov-like ansatz,

$$
\begin{aligned}
& \Phi_{\mathrm{tot}} \sim \xi\left(\begin{array}{cc}
0 & e^{i\left(\theta_{1}+\theta_{2}\right)} \\
1 & 0
\end{array}\right) \rightarrow \xi\left(\begin{array}{cc}
0 & e^{2 i \theta} \\
1 & 0
\end{array}\right), \\
& A_{\mathrm{tot}}=A_{(1)}+A_{(2)} \rightarrow-\frac{1}{2 g} \frac{\epsilon_{i j} x^{j}}{r^{2}} \sigma^{3}, \quad a_{\mathrm{tot}}=a_{(1)}+a_{(2)} \rightarrow-\frac{1}{e} \frac{\epsilon_{i j} x^{j}}{r^{2}}, \\
& \Psi_{\mathrm{tot}} \sim\left(\begin{array}{c}
a\left[e^{i \frac{\theta_{1}+\theta_{2}}{2}}+e^{i \frac{\theta_{1}-\theta_{2}}{2}} f_{1}\left(\theta_{1}-\theta_{2}-\pi\right)\right] \\
b
\end{array}\right) \rightarrow\left(\begin{array}{c}
a e^{i \theta} \\
b
\end{array}\right) .
\end{aligned}
$$


Then, the final state indicated after the arrows will be the doubly-wound Alice string configuration with a singly quantized U(1) magnetic flux and half-quantized SU(2) magnetic flux in eq. (3.14). In this case, the $\mathrm{SU}(2)$ color flux remains in the confined string. One may think that color is not confined. However, this color flux is screened and cannot be seen from the large distance by $\mathrm{AB}$ phases.

If we consider the deconfined phase, either of the two components of the doublet is zero, the $\mathbb{Z}_{4}$ symmetry is unbroken, and domain walls are not topologically supported. In this case, either of ANO string is free from an AB defect as can be seen in eq. (4.5), and consequently it is not confined (but the other is confined).

Comparing the vacuum manifolds for the deconfined and confined phases in eq. (2.23), one can understand that the confined phase does not admit a half $\mathrm{U}(1)$ winding (and therefore a half-quantized U(1) magnetic flux), while the deconfined phase does, consistent with the above discussion.

From the vacuum manifolds in eq. (2.33) for the second symmetry breaking in the opposite hierarchy, one can understand that in the confined phase strings are half-quantized strings corresponding to doubly-wound Alice strings, in contrast to the deconfined phase admitting 1/4-quantized strings corresponding to the Alice strings.

\subsection{Decay of Abrikosov-Nielsen-Olesen string and $\mathbb{Z}_{2}$ string}

Next, let us discuss the case of decay corresponding to figure 4(b).

An ANO vortex is attached by two AB defects in the confined phase as in eq. (4.10) and figure 3 (b). It decays and is split into a pair of Alice strings with the opposite $\mathrm{SU}(2)$ fluxes:

$$
\begin{aligned}
\Phi_{1}(r, \theta) & \sim \xi\left(\begin{array}{cc}
0 & e^{i \theta_{1}} \\
1 & 0
\end{array}\right)=\xi e^{i \frac{\theta_{1}}{2}} e^{+i \frac{\theta_{1}}{4} \sigma^{3}} \sigma^{1} e^{-i \frac{\theta_{1}}{4} \sigma^{3}}, \\
A_{1, i} & \sim-\frac{1}{4 g} \frac{\epsilon_{i j} x_{1}^{j}}{r_{1}^{2}} \sigma^{3}, \quad a_{1, i} \sim-\frac{1}{2 e} \frac{\epsilon_{i j} x_{1}^{j}}{r_{1}^{2}}, \\
\Psi_{1} & \sim\left(\begin{array}{c}
a e^{i \frac{\theta_{1}}{2}} f_{1}\left(\theta_{1}-\alpha_{1}\right) \\
b
\end{array}\right)
\end{aligned}
$$

and

$$
\begin{aligned}
\Phi_{2}(r, \theta) & \sim \xi\left(\begin{array}{cc}
0 & 1 \\
e^{i \theta_{2}} & 0
\end{array}\right)=\xi e^{i \frac{\theta_{2}}{2}} e^{-i \frac{\theta_{2}}{4} \sigma^{3}} \sigma^{1} e^{+i \frac{\theta_{2}}{4} \sigma^{3}}, \\
A_{2, i} & \sim+\frac{1}{4 g} \frac{\epsilon_{i j} x_{2}^{j}}{r_{2}^{2}} \sigma^{3}, \quad a_{2, i} \sim-\frac{1}{2 e} \frac{\epsilon_{i j} x_{2}^{j}}{r_{2}^{2}} \\
\Psi_{2} & \sim\left(\begin{array}{c}
a \\
b e^{i \frac{\theta}{2}} f_{2}\left(\theta-\alpha_{2}\right)
\end{array}\right) .
\end{aligned}
$$

Here, the coordinates are the same with figure 5 , and the configurations $\left(\Phi_{1}, A_{1}, a_{1}, \Psi_{1}\right)$ and $\left(\Phi_{2}, A_{2}, a_{2}, \Psi_{2}\right)$ denote vortices placed at $(a, 0)$ and $(-a, 0)$ as in figure $4(\mathrm{~b})$. These 
two vortices cannot be connected by an $\mathrm{AB}$ defect. Instead, two different $\mathrm{AB}$ defects are attached to each of them and pull the vortices to the opposite (or different) directions.

$\mathrm{A} \mathbb{Z}_{2}$ vortex is also attached by two $\mathrm{AB}$ defects in the confined phase as in eq. (4.11) and figure 3 (b). It also decays and is split into a pair of Alice string and anti-Alice string with the same $\mathrm{SU}(2)$ magnetic fluxes:

$$
\begin{aligned}
\Phi_{1}(r, \theta) & \sim \xi\left(\begin{array}{cc}
0 & e^{i \theta_{1}} \\
1 & 0
\end{array}\right)=\xi e^{i \frac{\theta_{1}}{2}} e^{+i \frac{\theta_{1}}{4} \sigma^{3}} \sigma^{1} e^{-i \frac{\theta_{1}}{4} \sigma^{3}}, \\
A_{1, i} & \sim-\frac{1}{4 g} \frac{\epsilon_{i j} x_{1}^{j}}{r_{1}^{2}} \sigma^{3}, \quad a_{1, i} \sim-\frac{1}{2 e} \frac{\epsilon_{i j} x_{1}^{j}}{r_{1}^{2}} \\
\Psi_{1} & \sim\left(\begin{array}{c}
a e^{i \frac{\theta_{1}}{2}} f_{1}\left(\theta_{1}-\alpha_{1}\right) \\
b
\end{array}\right)
\end{aligned}
$$

and

$$
\begin{aligned}
\Phi_{2}(r, \theta) & \sim \xi\left(\begin{array}{cc}
0 & 1 \\
e^{-i \theta_{2}} & 0
\end{array}\right)=\xi e^{-i \frac{\theta_{2}}{2}} e^{+i \frac{\theta_{2}}{4} \sigma^{3}} \sigma^{1} e^{-i \frac{\theta_{2}}{4} \sigma^{3}}, \\
A_{2, i} & \sim-\frac{1}{4 g} \frac{\epsilon_{i j} x_{2}^{j}}{r_{2}^{2}} \sigma^{3}, \quad a_{2, i} \sim+\frac{1}{2 e} \frac{\epsilon_{i j} x_{2}^{j}}{r_{2}^{2}} \\
\Psi_{2} & \sim\left(\begin{array}{c}
a \\
b e^{-i \frac{\theta_{2}}{2}} f_{2}\left(-\theta_{2}+\alpha_{2}\right)
\end{array}\right) .
\end{aligned}
$$

Again, the coordinates are the same with figure 5 , and the configurations $\left(\Phi_{1}, A_{1}, a_{1}, \Psi_{1}\right)$ and $\left(\Phi_{2}, A_{2}, a_{2}, \Psi_{2}\right)$ denote vortices placed at $(a, 0)$ and $(-a, 0)$ as in figure 5 . These two vortices are attached and pulled by the two different $\mathrm{AB}$ defects as in figure 4(b).

If we consider the deconfined phase in which either of the two components of the doublet is zero, only one $\mathrm{AB}$ defect is attached to the ANO (or $\mathbb{Z}_{2}$ ) string, and either of two Alice strings is free from an $\mathrm{AB}$ defect as can be seen in eq. (4.5). However, the decay of the ANO string and $\mathbb{Z}_{2}$ string always occurs since either of constituents is attached by an AB defect.

\subsection{Confinement of Alice monopoles: monopole mesons}

Here we turn to discuss that monopoles are also confined. We first discuss the deconfined phase in which one of components of the doublet fields is zero: $\Psi=(a, 0)^{T}$. Then, we point out that the same story holds for the confined phase as well.

First, let us consider a monopole on an Alice string. Let an Alice string be placed along the $z$ direction as in eq. (3.17), where and the $\mathrm{U}(1)$ modulus $\beta$ of the string winds once along it from $\beta=0$ at $z \rightarrow-\infty$ to $\beta=2 \pi$ at $z \rightarrow+\infty$. Then, there is one monopole on the string at $\beta=\pi$ as section 3.3. In the absence of the doublet VEV, the monopole charge density is spread over the string. However, it is not the case in the presence of the doublet VEV.

To see this, we consider a Wilson loop for the doublet encircling the string. We consider the doublet fields have a VEV in the second component and an Alice string has a winding in the upper-right component, as the first line eq. (4.5) with the upper sign. Then, the 


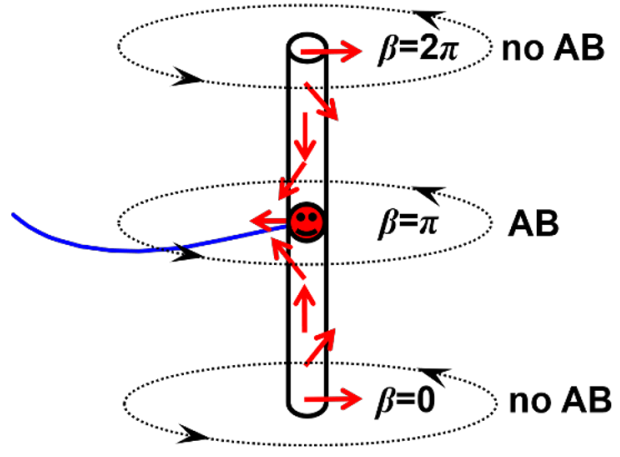

(a)

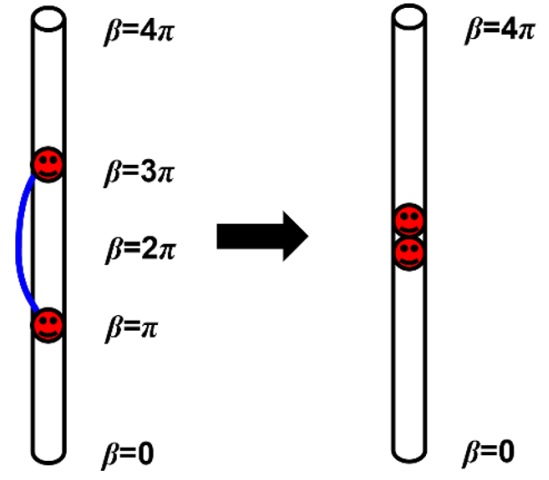

(b)

Figure 6. Monopole(s) on a string attached by AB defect(s) in the deconfined phase. (a) a single monopole on a string attached by an $\mathrm{AB}$ defect. Circles imply Wilson loops linking the string, which are trivial far from the monopole and exhibit an nontrivial AB phase around the monopole. (b) two monopoles on a string connected by an $\mathrm{AB}$ defect (left) and a monopole meson on a string (right).

Wilson loop is trivial for $\beta=0,2 \pi$ at $z= \pm \infty$. However, the Wilson loop encircling around the monopole $(\beta=\pi)$ is inevitably nontrivial, resulting in an AB defect (string) attached to the monopole as in figure 6 (a). In this sense, this can be regarded as a defect of the Wilson loop. The $\mathrm{U}(1)$ modulus $\beta$ is a sine-Gordon-like soliton along the string. If the $\mathrm{AB}$ defect is heavy enough compared with the tension of the Alice string, it will pull the string with constituting a $Y$-shape junction.

Next let us consider that the $\mathrm{U}(1)$ modulus $\beta$ of the Alice string winds twice along the string from $\beta=0$ at $z \rightarrow-\infty$ to $\beta=2 \pi$ at $z \rightarrow+\infty$. Then, there exist two monopoles, and each of them is attached by an AB defect string as in figure 6 (b) (left). Energetically, it is naturally to consider that these $\mathrm{AB}$ defects are connected, and the two monopoles are confined to be a monopole meson as in figure 6 (b) (right). Thus, the monopole confinement occurs on the Alice string.

Now, we are ready to discuss an isolated monopole. An isolated monopole can be obtained as a twisted Alice ring as discussed in section 3.3, which can be done by gluing the two ends of an Alice string in figure 6. Then, as shown in figure 7(a), an AB defect is attached on the monopole point on the Alice ring. The $U(1)$ modulus also becomes a sine-Gordon-like soliton on a ring. This monopole must be confined.

As the case of Alice strings, it can be confined with an anti-monopole. In that case, they are unstable and annihilate in pair. On the other hand, a monopole can be confined with another monopole as in figure 7(b). This configuration has the monopole charge two in total. There are two more possibilities of the monopole of the charge two. One is a double winding of the $\mathrm{U}(1)$ modulus $\beta$ along the Alice ring in figure $7(\mathrm{c})$, and the other is a singly twisted $\mathrm{U}(1)$ modulus $\beta$ along a ring of a doubly-wound Alice string as in figure $7(\mathrm{~d})$. Interestingly, the latter is not associated with any $\mathrm{AB}$ defect, since doubly-wound Alice strings do not have $\mathrm{AB}$ effects. It is a highly nontrivial question depending on parameter choices which configurations among figure $7(\mathrm{~b}),(\mathrm{c})$ and (d) is the most stable. 


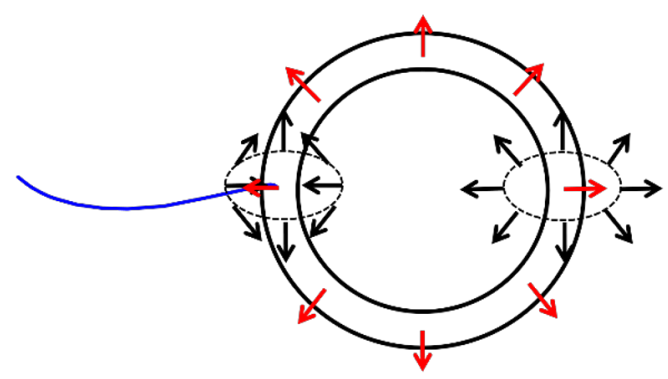

(a)

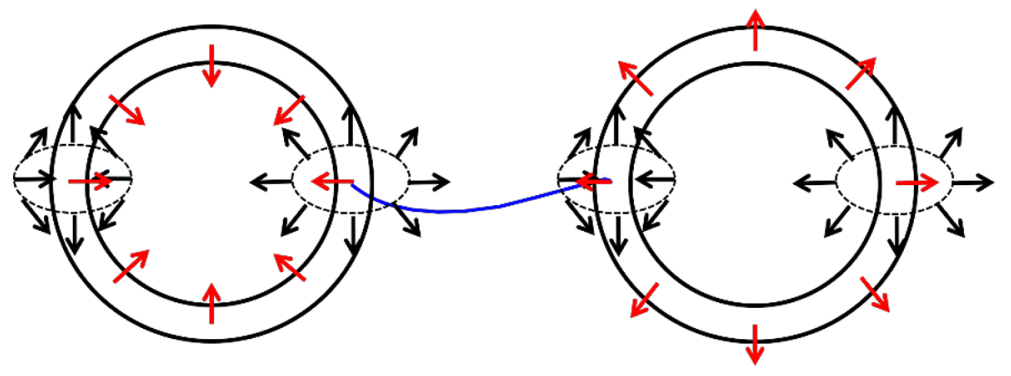

(b)

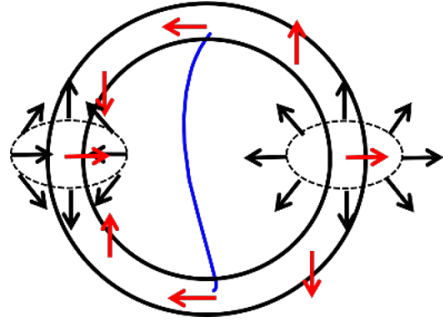

(c)

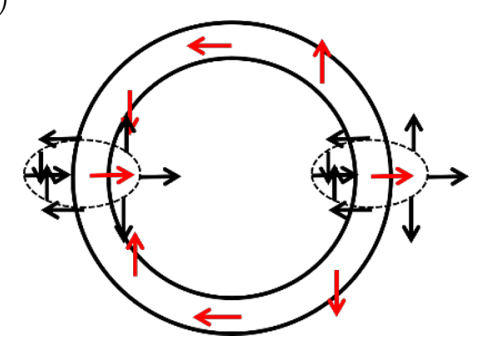

(d)

Figure 7. Confined monopoles in the deconfined phase. (a) A monopole of the charge one is attached by an $\mathrm{AB}$ defect. (b) Two monopoles of the charge one are connected by an $\mathrm{AB}$ defect. (c) A doubly-twisted Alice ring as a monopole of the charge two. (d) A singly twisted doublewinding Alice ring as a monopole of the charge two.

In summary, monopoles of a single (or odd) monopole charge is confined, and monopoles of an even monopole charge are not confined and are allowed to exist.

Let us discuss how monopoles look like in the deconfined phase. In this case, $A B$ defects appear around $\beta=0$ and $\beta=\pi$. Therefore, the $\mathrm{AB}$ defects disappear around $\beta= \pm \pi / 2$. Monopoles on a straight string become like figure 8(a). If we twist $\beta$ from once there appear two monopoles of the half charge, each of which is attached by two different $\mathrm{AB}$ defects corresponding to two components of the doublet. Therefore, in principle, only a half-monopole is possible to exist alone on a string. The effective theory for $\beta$ would be double sine-Gordon-like.

If we consider monopoles of charge two on a string, they are confined as in figure 8(b).

On the other hand, isolated monopoles can be constructed as twisted Alice rings as before. See figure 9. A single monopole is attached by two AB defects as in (a). This must 


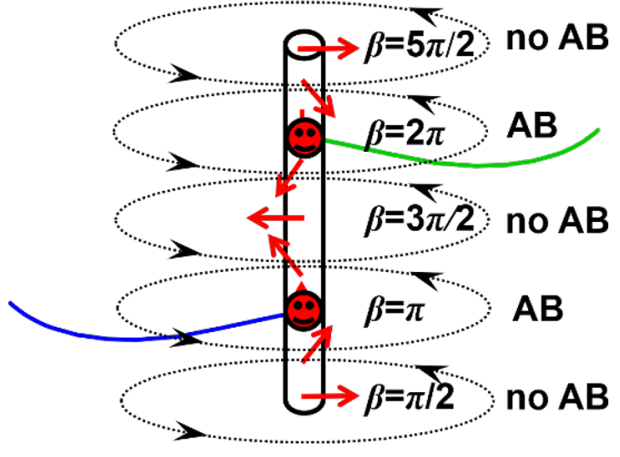

(a)

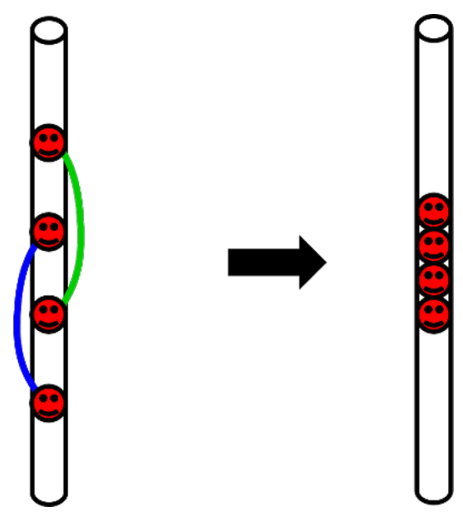

(b)

Figure 8. Monopoles on a string in the confined phase. (a) a single monopole on a string is split into two half monopoles, each of which is attached by a different $\mathrm{AB}$ defect. (b) two monopoles or four half monopoles on a string connected by the two different $\mathrm{AB}$ defects (left), and a monopole meson on a string (right).

be confined as either (b) or (c): two single monopoles are connected by two AB defects as in (b), and a doubly-twisted Alice ring have two AB defects connecting two sets of antipodal points of the ring as in (c).

\section{$5 \quad$ Summary and discussion}

We have studied an $\mathrm{SU}(2) \times \mathrm{U}(1)$ gauge theory with charged triplet complex scalar fields admitting Alice strings and Alice monopoles, and introduced the charged doublet scalar fields receiving nontrivial AB phases around the Alice string. An ANO string and a $\mathbb{Z}_{2}$ string carry unit U(1) magnetic flux and a half SU(2) magnetic flux, respectively. First, we have studied the vacua in the presence of the doublet VEVs. The unbroken symmetry is $\mathbb{Z}_{4}$ if one of VEVs is zero (the deconfined phase), and it is $\mathbb{Z}_{2}$ if both VEVs are nonzero (the confined phase). The Alice string carries a half $\mathrm{U}(1)$ magnetic flux and $1 / 4 \mathrm{SU}(2)$ magnetic flux taking a value in a linear combination of $\sigma^{2}$ and $\sigma^{3}$ (when the VEV of the triplet is $\sigma^{1}$ ). In the absence of a doublet VEV, the Alice string is deconfined in the sense that the color flux can be seen by AB phases of some fields (like our doublet fields) encircling the string. When the doublet field develops VEVs in the presence of a single Alice string, the bulksoliton moduli locking occurs; the vacuum moduli are locked with the U(1) modulus of the Alice string. The deconfined phase is realized to eliminate a domain wall called an AB defect responsible for single-valuedness of the doublet fields. On the other hand, in the confined phase, the Alice string is inevitably attached by an $\mathrm{AB}$ defect. Consequently, it is confined with either an anti-Alice string or the same type of an Alice string. In the former case, the pair annihilates. In the latter case, the pair forms a stable doubly-wound Alice string having a color magnetic flux inside the core, but the color cannot be seen at large distance by $\mathrm{AB}$ phases and so the color confinement occurs. We have referred these phases without and with doublet VEVs as the deconfined and confined phases, respectively. The results are also consistent with the vacuum manifold in eq. (2.12) admitting 1/4 quantized $\mathrm{SU}(2)$ fluxes for 


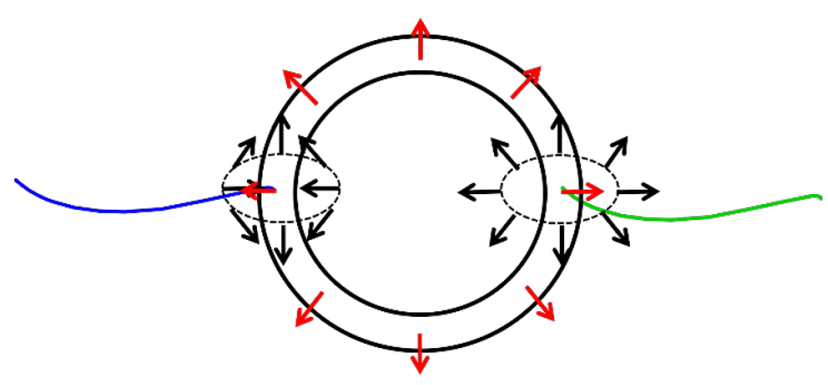

(a)

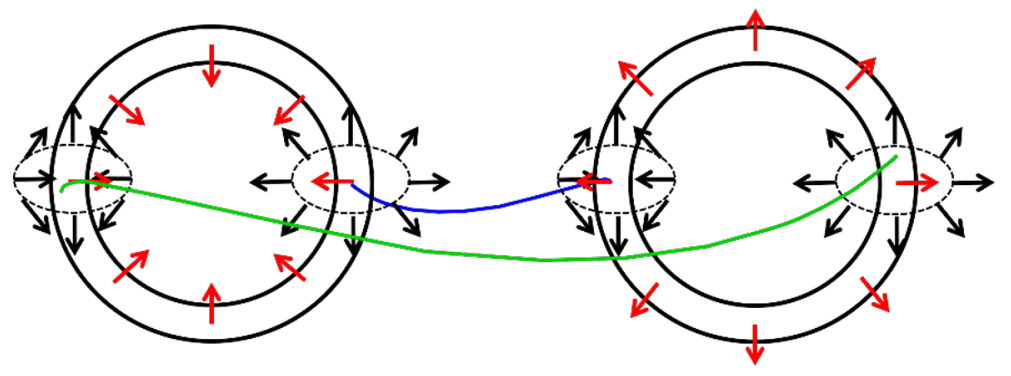

(b)

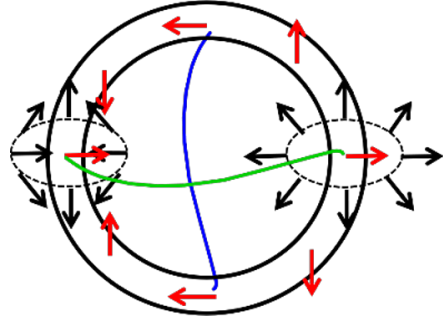

(c)

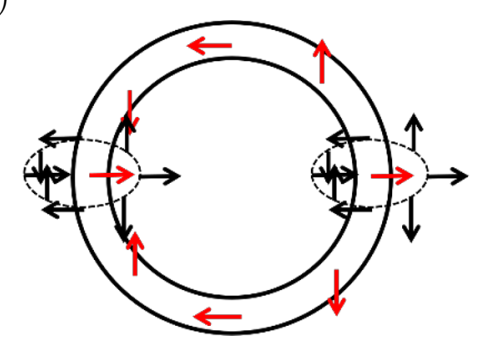

(d)

Figure 9. Confined monopoles in the confined phase. (a) A monopole of the charge one is attached by an $\mathrm{AB}$ defect. (b) Two monopoles of the charge one are connected by an $\mathrm{AB}$ defect. (c) A doublytwisted Alice ring as a monopole of the charge two. (d) A singly twisted double-winding Alice ring as a monopole of the charge two.

the deconfined phase, and the vacuum manifold in the confined phase in eq. (2.23) admitting only 1/2 quantized SU(2) fluxes for the confined phase. In the scheme that the doublet field develops VEVs at high energy, the vacuum manifolds in eq. (2.33) for the second symmetry breakings by the triplet field at low energy imply that the deconfined and confined phases allow $1 / 4$ and $1 / 2$ quantized $W_{3}$ magnetic fluxes, which are a single Alice string and doubly wound Alice string, respectively, implying the confinement in the confined phase. We also have shown that the ANO string and $\mathbb{Z}_{2}$ string decay into two Alice strings once the doublet field develops VEVs. Furthermore, a monopole in this theory can be constructed as a closed Alice string with the U(1) modulus twisted once, and we have found that when the doublet field develops VEVs, monopoles are confined to mesons of the monopole charge two.

When a charged particle encircles an Alice string in the absence of the doublet VEVs, its electric charge is flipped. This is an example of topological obstructions. In addition to 
this, when a monopole encircles an Alice string, a monopole charge is also flipped, which is an example of topological influence (see ref. [53] for a global analogue of this phenomenon). When the doublet field develops VEVs, Alice strings are confined to doubly-wound Alice strings and monopoles are confined to monopole mesons as discussed in this paper. In this case, it seems that the topological obstructions and topological influence disappear. This suggests that deconfinement and confinement phases may be characterized by the presence and absence, respectively of the topological obstruction and topological influence.

Several discussions are addressed here.

One of the most important questions may be whether the confined and deconfined phases are separated by a phase transtion. In order to investigate this problem, one needs to study higher-form global symmetries [69]. Higher-fom symmetries for the conventional Alice strings were studied in ref. [70]. In our case, it may be the case that there is a certain magnetic 2-form symmetry in our model and the breaking of this symmetry may separate the confined and deconfined phases by a phase transition. This is under investigation [71].

If we turn off the $\mathrm{U}(1)$ gauge coupling, $e=0$, and regard this global $\mathrm{U}(1)$ symmetry as the Peccei-Quinn symmetry for axions, our model becomes an axion dark matter model in refs. [60,61]. After chiral symmetry breaking, an axion string is attached by two axion domain walls and the domain wall number is two in the model. When the domain wall number is more than one, usually there is a domain wall problem (if domain walls cannot decay and dominate Universe) [72]. In our case, one axion string attached by two domain walls can decay into two Alice strings each of which is attached by only one domain wall, thereby free from the domain wall problem. In ref. [60], the doublet scalar field was also considered. This doublet field receives an $\mathrm{AB}$ phase as discussed in the present paper. If the doublet develops a VEV, one axion string (corresponding to ANO string in this paper) can decay into two Alice strings, thereby a domain wall problem does not occur even before chiral symmetry breaking.

The third homotopy group for the first symmetry breaking by the triplet in eq. (2.13) implies the existence of gauged Hopfions (for a global analogues of the same breaking, see ref. [78]). The second homotopy group for the second symmetry breaking by the doublet in eq. (2.22) implies that there are another type of vortices in the both deconfined and confined phases. Studying these topological objects remains as a future problem.

Fermions receiving non-trivial $\mathrm{AB}$ phases can contribute to $\mathrm{AB}$ defects if they form pair condensations. In fact, such an example can be found in dense QCD [37], in which an $\mathrm{SU}(3)$ generalization of our model was discussed.

After completion of this paper, the author was informed that a phenemenon similar to the bulk-soliton moduli locking was also discussed in refs. [79-81].

\section{Acknowledgments}

The author thanks Chandrasekhar Chatterjee for discussion in the previous works and at the early stage of this work, and Yoshimasa Hidaka and Ryo Yokokura for discussion about higher-form symmetries. This work is supported in part by JSPS Grant-in-Aid for Scientific Research (KAKENHI Grant No. 18H01217). 


\section{A Complex symmetric tensor}

Here, we summarize an equivalence between a complex adjoint scalar field and a complex symmetric tensor scalar field.

A complex adjoint scalar field considered in this paper

$$
\Phi=\sum_{\alpha=1}^{3} \Phi^{\alpha} \sigma^{\alpha}
$$

transforms as

$$
\Phi \rightarrow \Phi^{\prime}=e^{i \alpha} g \Phi g^{-1}, \quad\left(e^{i \alpha}, g\right) \in \mathrm{U}(1)_{Y} \times \mathrm{SU}(2)_{W} .
$$

On the other hand, a complex $2 \times 2$ symmetric tensor scalar field

$$
M=M_{0} \mathbf{1}_{2}+M_{1} \sigma^{1}+M_{3} \sigma^{3}
$$

transforms as

$$
M \rightarrow M^{\prime}=e^{i \alpha} g M g^{T}, \quad\left(e^{i \alpha}, g\right) \in \mathrm{U}(1)_{Y} \times \mathrm{SU}(2)_{W} .
$$

Both fields have three complex scalar degrees of freedom.

From the relations

$$
\begin{aligned}
\left(+i \sigma^{2}\right)\left(\mathbf{1}_{2}, \vec{\sigma}^{T}\right)\left(-i \sigma^{2}\right) & =\left(\mathbf{1}_{2},-\vec{\sigma}\right) \\
\left(i \sigma^{2}\right) g^{T}\left(-i \sigma^{2}\right) & =g^{-1}, \quad g \in \mathrm{SU}(2),
\end{aligned}
$$

we can easily see that $M\left(-i \sigma^{2}\right)$ in fact transforms as an adjoint representation, thus implying the relation between them,

$$
\begin{aligned}
\Phi=M\left(-i \sigma^{2}\right) & =-i M_{0} \sigma^{2}+M_{1} \sigma^{3}-M_{3} \sigma^{1}, \\
\left(\Phi^{1}, \Phi^{2}, \Phi^{3}\right) & =\left(-M_{3},-i M_{0}, M_{1}\right) .
\end{aligned}
$$

From the coefficient $i$ in the second component, one can understand that this relation holds only between complex scalar fields but not between real scalar fields.

The vacuum $\Phi=\Phi^{1} \sigma^{1}$ in eq. (2.6) considered in this paper corresponds to $M=M_{3} \sigma^{3}$. The unbroken subgroup of the $M=\xi \sigma^{3}$ is $H=\left(\mathbb{Z}_{4}\right)_{Y+W_{2,3}} \ltimes \mathrm{SO}(2)_{W_{1}}$ with the $\operatorname{SO}(2)$ part generated by $\sigma_{1}$, which of course does not depend on a choice of representation $\Phi$ or $M$, equivalent to each other.

In terms of the symmetric tensor $M$, the ANO and $\mathbb{Z}_{2}$ strings can be written as

$$
\begin{aligned}
& M(r, \theta) \sim \xi\left(\begin{array}{cc}
e^{i \theta} & 0 \\
0 & -e^{i \theta}
\end{array}\right)=\xi e^{i \theta} \sigma^{3}, \\
& M(r, \theta) \sim \xi\left(\begin{array}{cc}
e^{i \theta} & 0 \\
0 & -e^{-i \theta}
\end{array}\right)=\xi e^{i \frac{\theta}{2} \sigma^{3}} \sigma^{3} e^{i \frac{\theta}{2} \sigma^{3}},
\end{aligned}
$$

with the gauge field configurations in eqs. (3.1) and (3.2), respectively. 
The Alice strings corresponding to eq. (3.3) and (3.4) can be written as

$$
\begin{aligned}
& M(r, \theta) \sim \xi\left(\begin{array}{cc}
e^{i \theta} & 0 \\
0 & 1
\end{array}\right)=\xi e^{i \frac{\theta}{2}}\left(\begin{array}{cc}
e^{+i \frac{\theta}{2}} & 0 \\
0 & -e^{-i \frac{\theta}{2}}
\end{array}\right)=\xi e^{i \frac{\theta}{2}} e^{+i \frac{\theta}{4} \sigma^{3}} \sigma^{3} e^{+i \frac{\theta}{4} \sigma^{3}}, \\
& M(r, \theta) \sim \xi\left(\begin{array}{cc}
1 & 0 \\
0 & -e^{i \theta}
\end{array}\right)=\xi e^{i \frac{\theta}{2}}\left(\begin{array}{cc}
e^{-i \frac{\theta}{2}} & 0 \\
0 & -e^{+i \frac{\theta}{2}}
\end{array}\right)=\xi e^{i \frac{\theta}{2}} e^{-i \frac{\theta}{4} \sigma^{3}} \sigma^{3} e^{-i \frac{\theta}{4} \sigma^{3}},
\end{aligned}
$$

with the same gauge field configurations in eq. (3.3) and (3.4), respectively, while their anti-strings in eqs. (3.7) and (3.8) can be written as

$$
\begin{aligned}
& M(r, \theta) \sim \xi\left(\begin{array}{cc}
1 & 0 \\
0 & -e^{-i \theta}
\end{array}\right)=\xi e^{-i \frac{\theta}{2}}\left(\begin{array}{cc}
e^{+i \frac{\theta}{2}} & 0 \\
0 & -e^{-i \frac{\theta}{2}}
\end{array}\right)=\xi e^{-i \frac{\theta}{2}} e^{+i \frac{\theta}{4} \sigma^{3}} \sigma^{3} e^{+i \frac{\theta}{4} \sigma^{3}}, \\
& M(r, \theta) \sim \xi\left(\begin{array}{cc}
e^{-i \theta} & 0 \\
0 & 1
\end{array}\right)=\xi e^{-i \frac{\theta}{2}}\left(\begin{array}{cc}
e^{-i \frac{\theta}{2}} & 0 \\
0 & -e^{+i \frac{\theta}{2}}
\end{array}\right)=\xi e^{-i \frac{\theta}{2}} e^{-i \frac{\theta}{4} \sigma^{3}} \sigma^{3} e^{-i \frac{\theta}{4} \sigma^{3}},
\end{aligned}
$$

respectively, with the same gauge field configurations.

The doubly-wound Alice strings corresponding to eq. (3.14) and (3.15) can be written as

$$
\begin{aligned}
& M(r, \theta) \sim \xi\left(\begin{array}{cc}
e^{2 i \theta} & 0 \\
0 & 1
\end{array}\right)=\xi e^{i \theta}\left(\begin{array}{cc}
e^{+i \theta} & 0 \\
0 & -e^{-i \theta}
\end{array}\right)=\xi e^{i \theta} e^{+i \frac{\theta}{2} \sigma^{3}} \sigma^{3} e^{+i \frac{\theta}{2} \sigma^{3}}, \\
& M(r, \theta) \sim \xi\left(\begin{array}{cc}
1 & 0 \\
0 & -e^{2 i \theta}
\end{array}\right)=\xi e^{i \theta}\left(\begin{array}{cc}
e^{-i \theta} & 0 \\
0 & -e^{+i \theta}
\end{array}\right)=\xi e^{i \theta} e^{-i \frac{\theta}{2} \sigma^{3}} \sigma^{3} e^{-i \frac{\theta}{2} \sigma^{3}}
\end{aligned}
$$

with the same gauge field configurations in eq. (3.14) and (3.15), respectively.

\section{B Alice string configurations}

Here, we briefly summarize BPS Alice strings $[57,58]$ in the absence of the doublet field. setting all the scalar couplings to be zero except $\lambda_{g}$ and $\lambda_{e}$. The static Hamiltonian density is given by

$$
H=\int d^{3} x\left[\frac{1}{2} \operatorname{Tr} F_{i j} F^{i j}+\frac{1}{4} f_{i j} f^{i j}+\operatorname{Tr}\left|D_{i} \Phi\right|^{2}+\frac{\lambda_{g}}{4} \operatorname{Tr}\left[\Phi, \Phi^{\dagger}\right]^{2}+\frac{\lambda_{e}}{2}\left(\operatorname{Tr} \Phi \Phi^{\dagger}-2 \xi^{2}\right)^{2}\right] .
$$

At the critical couplings $\lambda_{e}=e^{2}$ and $\lambda_{g}=g^{2}$, the Bogomol'nyi completion of the tension can be performed as

$$
\begin{aligned}
\mathcal{T} & =\int d^{2} x\left[\operatorname{Tr}\left[F_{12} \pm \frac{g}{2}\left[\Phi, \Phi^{\dagger}\right]\right]^{2}+\operatorname{Tr}\left|\mathcal{D}_{ \pm} \Phi\right|^{2}+\frac{1}{2}\left[f_{12} \pm e\left(\operatorname{Tr} \Phi \Phi^{\dagger}-2 \xi^{2}\right)\right]^{2} \pm 2 e f_{12} \xi^{2}\right] \\
& \geq 2 e \xi^{2}\left|\int d^{2} x f_{12}\right|=2 \pi \xi^{2},
\end{aligned}
$$

with $\mathcal{D}_{ \pm} \equiv \frac{D_{1} \pm i D_{2}}{2}$. The equality is achieved if the BPS equations are satisfied:

$$
\begin{aligned}
F_{12} \pm \frac{g}{2}\left[\Phi, \Phi^{\dagger}\right] & =0 \\
\mathcal{D}_{ \pm} \Phi & =0 \\
f_{12} \pm e\left(\operatorname{Tr} \Phi \Phi^{\dagger}-2 \xi^{2}\right) & =0 .
\end{aligned}
$$




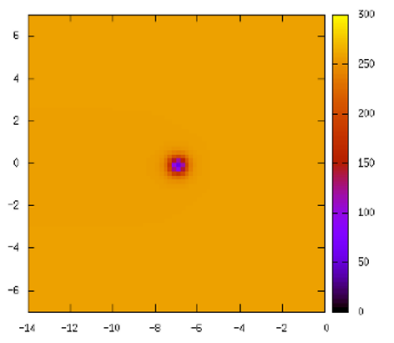

(a)

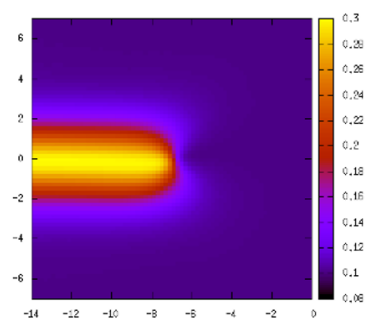

(b)

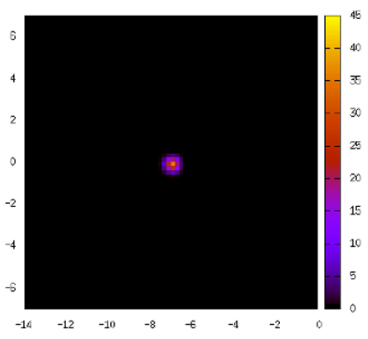

(c)

Figure 10. A full 2D numerical simulation for an Alice string confined by a soliton (figures taken from [59]). (a) The gauge invariant $4 \operatorname{Tr} \Phi^{2} \operatorname{Tr} \Phi^{\dagger^{2}} \sim \xi^{4} f(r)^{2} g(r)^{2}$ vanishing at the center of the Alice string. (b) The interaction potential $V_{\text {int }}^{(1)}+\frac{2 \xi m^{2}}{\lambda_{\psi}}$, in which the domain wall is clearly visible. (c) The flux squared $\operatorname{Tr} F_{12}^{2}=\sum_{a=1}^{3} F_{12}^{a}{ }^{2}$. The parameter choices are $\xi=0.1, e=0.5, g=1, \mu^{(1)}=$ $0.1, \lambda_{\psi}=1, m=0.1, \lambda_{e}=2, \lambda_{g}=1$.

Alice string solution can be constructed by inserting profile functions $f_{1}(r), f_{2}(r), A(r)$ and $a(r)$ into the asymptotic form in eq. (3.3):

$$
\begin{aligned}
\Phi(r, \theta) & =\xi\left(\begin{array}{cc}
0 & f_{1}(r) e^{i \theta} \\
f_{2}(r) & 0
\end{array}\right), \\
A_{i}(r, \varphi) & =-\frac{1}{4 g} \frac{\epsilon_{i j} x_{j}}{r^{2}} \sigma^{3} A(r), \quad a_{i}(r, \theta)=-\frac{1}{2 e} \frac{\epsilon_{i j} x_{j}}{r^{2}} a(r),
\end{aligned}
$$

where $(r, \theta)$ are radial and angular coordinates of the two dimensional space, respectively. The profile functions depend only on the radial coordinate and can be solved numerically with the boundary conditions $f_{1}(0)=f_{2}^{\prime}(0)=0, \quad f_{1}(\infty)=f_{2}(\infty)=1, A(0)=a(0)=$ 0, $A(\infty)=a(\infty)=1 .{ }^{17}$ The numerical solution for the BPS equation (B.3) can be found in ref. [57].

Once the doublet field develops VEVs, only 2D full numerical solution of an Alice string attached by an $\mathrm{AB}$ defect is available [59], see figure 10 .

Open Access. This article is distributed under the terms of the Creative Commons Attribution License (CC-BY 4.0), which permits any use, distribution and reproduction in any medium, provided the original author(s) and source are credited.

\section{References}

[1] Y. Aharonov and D. Bohm, Significance of electromagnetic potentials in the quantum theory, Phys. Rev. 115 (1959) 485 [InSPIRE].

[2] A. Tonomura et al., Observation of Aharonov-Bohm effect by electron holography, Phys. Rev. Lett. 48 (1982) 1443.

[3] A. Tonomura et al., Evidence for Aharonov-Bohm effect with magnetic field completely shielded from electron wave, Phys. Rev. Lett. 56 (1986) 792.

\footnotetext{
${ }^{17}$ These profile functions eventually satisfy the same equations with those for a non-Abelian vortex in $\mathrm{U}(N)$ gauge theory coupled with $N$ Higgs scalar fields in the fundamental representation [12-20].
} 
[4] M.G. Alford and F. Wilczek, Aharonov-Bohm interaction of cosmic strings with matter, Phys. Rev. Lett. 62 (1989) 1071 [INSPIRE].

[5] A. Vilenkin, Cosmic string dynamics with friction, Phys. Rev. D 43 (1991) 1060 [InSPIRE].

[6] J. March-Russell, J. Preskill and F. Wilczek, Internal frame dragging and a global analog of the Aharonov-Bohm effect, Phys. Rev. Lett. 68 (1992) 2567 [hep-th/9112054] [INSPIRE].

[7] J. Polchinski, Open heterotic strings, JHEP 09 (2006) 082 [hep-th/0510033] [INSPIRE].

[8] J.A. Harvey and A.B. Royston, Localized modes at a D-brane-O-plane intersection and heterotic Alice atrings, JHEP 04 (2008) 018 [arXiv:0709.1482] [INSPIRE].

[9] J.A. Harvey and A.B. Royston, Gauge/gravity duality with a chiral $N=(0,8)$ string defect, JHEP 08 (2008) 006 [arXiv:0804.2854] [INSPIRE].

[10] Y. Ookouchi, Discrete gauge symmetry and Aharonov-Bohm radiation in string theory, JHEP 01 (2014) 049 [arXiv: 1310.4026] [INSPIRE].

[11] T. Okada and Y. Sakatani, Defect branes as Alice strings, JHEP 03 (2015) 131 [arXiv: 1411.1043] [INSPIRE].

[12] A. Hanany and D. Tong, Vortices, instantons and branes, JHEP 07 (2003) 037 [hep-th/0306150] [INSPIRE].

[13] R. Auzzi, S. Bolognesi, J. Evslin, K. Konishi and A. Yung, Non-Abelian superconductors: vortices and confinement in $N=2$ SQCD, Nucl. Phys. B 673 (2003) 187 [hep-th/0307287] [INSPIRE].

[14] M. Eto, Y. Isozumi, M. Nitta, K. Ohashi and N. Sakai, Instantons in the Higgs phase, Phys. Rev. D 72 (2005) 025011 [hep-th/0412048] [INSPIRE].

[15] M. Eto, Y. Isozumi, M. Nitta, K. Ohashi and N. Sakai, Moduli space of non-Abelian vortices, Phys. Rev. Lett. 96 (2006) 161601 [hep-th/0511088] [INSPIRE].

[16] M. Eto et al., Non-Abelian vortices of higher winding numbers, Phys. Rev. D 74 (2006) 065021 [hep-th/0607070] [INSPIRE].

[17] D. Tong, TASI lectures on solitons: instantons, monopoles, vortices and kinks, in Theoretical Advanced Study Institute in Elementary Particle Physics: many dimensions of string theory, (2005) [hep-th/0509216] [INSPIRE].

[18] M. Eto, Y. Isozumi, M. Nitta, K. Ohashi and N. Sakai, Solitons in the Higgs phase: the moduli matrix approach, J. Phys. A 39 (2006) R315 [hep-th/0602170] [INSPIRE].

[19] M. Shifman and A. Yung, Supersymmetric solitons and how they help us understand non-Abelian gauge theories, Rev. Mod. Phys. 79 (2007) 1139 [hep-th/0703267] [INSPIRE].

[20] M. Shifman and A. Yung, Supersymmetric solitons, Cambridge University Press, Cambridge, U.K. (2009) [INSPIRE].

[21] J. Evslin, K. Konishi, M. Nitta, K. Ohashi and W. Vinci, Non-Abelian vortices with an Aharonov-Bohm effect, JHEP 01 (2014) 086 [arXiv:1310.1224] [INSPIRE].

[22] S. Bolognesi, C. Chatterjee and K. Konishi, Non-Abelian vortices, large winding limits and Aharonov-Bohm effects, JHEP 04 (2015) 143 [arXiv: 1503.00517] [INSPIRE].

[23] S. Bolognesi, C. Chatterjee, J. Evslin, K. Konishi, K. Ohashi and L. Seveso, Geometry and dynamics of a coupled 4D-2D quantum field theory, JHEP 01 (2016) 075 [arXiv: 1509.04061] [INSPIRE]. 
[24] M.G. Alford and A. Sedrakian, Color-magnetic flux tubes in quark matter cores of neutron stars, J. Phys. G 37 (2010) 075202 [arXiv:1001.3346] [INSPIRE].

[25] A.P. Balachandran, S. Digal and T. Matsuura, Semi-superfluid strings in high density QCD, Phys. Rev. D 73 (2006) 074009 [hep-ph/0509276] [INSPIRE].

[26] E. Nakano, M. Nitta and T. Matsuura, Non-Abelian strings in high density QCD: zero modes and interactions, Phys. Rev. D 78 (2008) 045002 [arXiv:0708.4096] [INSPIRE].

[27] M. Eto, E. Nakano and M. Nitta, Effective world-sheet theory of color magnetic flux tubes in dense QCD, Phys. Rev. D 80 (2009) 125011 [arXiv:0908.4470] [INSPIRE].

[28] M. Eto and M. Nitta, Color magnetic flux tubes in dense QCD, Phys. Rev. D 80 (2009) 125007 [arXiv: 0907.1278] [INSPIRE].

[29] M. Eto, M. Nitta and N. Yamamoto, Instabilities of non-Abelian vortices in dense QCD, Phys. Rev. Lett. 104 (2010) 161601 [arXiv:0912.1352] [INSPIRE].

[30] M. Eto, Y. Hirono, M. Nitta and S. Yasui, Vortices and other topological solitons in dense quark matter, PTEP 2014 (2014) 012D01 [arXiv:1308.1535] [INSPIRE].

[31] C. Chatterjee and M. Nitta, Aharonov-Bohm phase in high density quark matter, Phys. Rev. D 93 (2016) 065050 [arXiv: 1512.06603] [INSPIRE].

[32] A. Cherman, S. Sen and L.G. Yaffe, Anyonic particle-vortex statistics and the nature of dense quark matter, Phys. Rev. D 100 (2019) 034015 [arXiv: 1808.04827] [INSPIRE].

[33] C. Chatterjee, M. Nitta and S. Yasui, Quark-hadron continuity under rotation: vortex continuity or boojum?, Phys. Rev. D 99 (2019) 034001 [arXiv: 1806.09291] [InSPIRE].

[34] C. Chatterjee, M. Nitta and S. Yasui, Quark-hadron crossover with vortices, JPS Conf. Proc. 26 (2019) 024030 [arXiv:1902.00156] [INSPIRE].

[35] Y. Hirono and Y. Tanizaki, Quark-hadron continuity beyond the Ginzburg-Landau paradigm, Phys. Rev. Lett. 122 (2019) 212001 [arXiv:1811.10608] [INSPIRE].

[36] Y. Hirono and Y. Tanizaki, Effective gauge theories of superfluidity with topological order, JHEP 07 (2019) 062 [arXiv:1904.08570] [INSPIRE].

[37] Y. Fujimoto and M. Nitta, Non-Abelian Alice strings in two-flavor dense QCD, Phys. Rev. D 103 (2021) 054002 [arXiv: 2011.09947] [INSPIRE].

[38] A. Schwarz, Field theories with no local conservation of the electric charge, Nucl. Phys. B 208 (1982) 141.

[39] J.E. Kiskis, Disconnected gauge groups and the global violation of charge conservation, Phys. Rev. D 17 (1978) 3196 [INSPIRE].

[40] J. Striet and F.A. Bais, Simple models with Alice fluxes, Phys. Lett. B 497 (2000) 172 [hep-th/0010236] [INSPIRE].

[41] F.A. Bais and J. Striet, On a core instability of 't Hooft-Polyakov monopoles, Phys. Lett. B 540 (2002) 319 [hep-th/0205152] [INSPIRE].

[42] J. Striet and F.A. Bais, More on core instabilities of magnetic monopoles, JHEP 06 (2003) 022 [hep-th/0304189] [INSPIRE].

[43] K.M. Benson and T. Imbo, Topologically Alice strings and monopoles, Phys. Rev. D 70 (2004) 025005 [hep-th/0407001] [INSPIRE]. 
[44] M.G. Alford, K. Benson, S.R. Coleman, J. March-Russell and F. Wilczek, The interactions and excitations of non-Abelian vortices, Phys. Rev. Lett. 64 (1990) 1632 [Erratum ibid. 65 (1990) 668] [INSPIRE].

[45] M.G. Alford, K. Benson, S.R. Coleman, J. March-Russell and F. Wilczek, Zero modes of non-Abelian vortices, Nucl. Phys. B 349 (1991) 414 [InSPIRE].

[46] M.G. Alford, K.-M. Lee, J. March-Russell and J. Preskill, Quantum field theory of non-Abelian strings and vortices, Nucl. Phys. B 384 (1992) 251 [hep-th/9112038] [INSPIRE].

[47] J. Preskill and L.M. Krauss, Local discrete symmetry and quantum mechanical hair, Nucl. Phys. B 341 (1990) 50 [inSPIRE].

[48] M. Bucher, H.-K. Lo and J. Preskill, Topological approach to Alice electrodynamics, Nucl. Phys. B 386 (1992) 3 [hep-th/9112039] [INSPIRE].

[49] H.-K. Lo and J. Preskill, Non-Abelian vortices and non-Abelian statistics, Phys. Rev. D 48 (1993) 4821 [hep-th/9306006] [INSPIRE].

[50] M. Bucher and A. Goldhaber, $\mathrm{SO}(10)$ cosmic strings and $\mathrm{SU}(3)$-color Cheshire charge, Phys. Rev. D 49 (1994) 4167 [hep-ph/9310262] [INSPIRE].

[51] U. Leonhardt and G.E. Volovik, How to create Alice string (half quantum vortex) in a vector Bose-Einstein condensate, Pisma Zh. Eksp. Teor. Fiz. 72 (2000) 66 [JETP Lett. 72 (2000) 46] [cond-mat/0003428] [INSPIRE].

[52] J. Ruostekoski and J.R. Anglin, Monopole core instability and Alice rings in spinor Bose-Einstein condensates, Phys. Rev. Lett. 91 (2003) 190402 [Erratum ibid. 97 (2006) 069902] [cond-mat/0307651] [INSPIRE].

[53] S. Kobayashi, M. Kobayashi, Y. Kawaguchi, M. Nitta and M. Ueda, Abe homotopy classification of topological excitations under the topological influence of vortices, Nucl. Phys. B 856 (2012) 577 [arXiv:1110.1478] [INSPIRE].

[54] Y. Kawaguchi and M. Ueda, Spinor Bose-Einstein condensates, Phys. Rept. 520 (2012) 253 [INSPIRE].

[55] E.B. Bogomolny, Stability of classical solutions, Sov. J. Nucl. Phys. 24 (1976) 449 [Yad. Fiz. 24 (1976) 861] [INSPIRE].

[56] M.K. Prasad and C.M. Sommerfield, An exact classical solution for the 't Hooft monopole and the Julia-Zee dyon, Phys. Rev. Lett. 35 (1975) 760 [InSPIRE].

[57] C. Chatterjee and M. Nitta, BPS Alice strings, JHEP 09 (2017) 046 [arXiv:1703.08971] [INSPIRE].

[58] C. Chatterjee and M. Nitta, The effective action of a BPS Alice string, Eur. Phys. J. C 77 (2017) 809 [arXiv: 1706.10212] [INSPIRE].

[59] C. Chatterjee and M. Nitta, Aharonov-Bohm defects, Phys. Rev. D 101 (2020) 085002 [arXiv: 1905.01884] [INSPIRE].

[60] R. Sato, F. Takahashi and M. Yamada, Unified origin of axion and monopole dark matter, and solution to the domain-wall problem, Phys. Rev. D 98 (2018) 043535 [arXiv: 1805.10533] [INSPIRE].

[61] C. Chatterjee, T. Higaki and M. Nitta, Note on a solution to domain wall problem with the Lazarides-Shafi mechanism in axion dark matter models, Phys. Rev. D 101 (2020) 075026 [arXiv: 1903.11753] [INSPIRE]. 
[62] P. Materne et al., Solitonic spin-liquid state due to the violation of the Lifshitz condition in $F_{1+y}$ Te, Phys. Rev. Lett. 115 (2015) 177203 [arXiv:1510.07486].

[63] A.A. Abrikosov, On the magnetic properties of superconductors of the second group, Sov. Phys. JETP 5 (1957) 1174 [Zh. Eksp. Teor. Fiz. 32 (1957) 1442] [INSPIRE].

[64] H.B. Nielsen and P. Olesen, Vortex line models for dual strings, Nucl. Phys. B 61 (1973) 45 [INSPIRE].

[65] H. Georgi and M. Machacek, Doubly charged Higgs bosons, Nucl. Phys. B 262 (1985) 463 [INSPIRE].

[66] C. Chatterjee, M. Kurachi and M. Nitta, Topological defects in the Georgi-Machacek model, Phys. Rev. D 97 (2018) 115010 [arXiv:1801.10469] [INSPIRE].

[67] T.W.B. Kibble, G. Lazarides and Q. Shafi, Walls bounded by strings, Phys. Rev. D 26 (1982) 435 [INSPIRE].

[68] A. Vilenkin and A.E. Everett, Cosmic strings and domain walls in models with Goldstone and pseudo-Goldstone bosons, Phys. Rev. Lett. 48 (1982) 1867 [InSPIRE].

[69] D. Gaiotto, A. Kapustin, N. Seiberg and B. Willett, Generalized global symmetries, JHEP 02 (2015) 172 [arXiv: 1412.5148] [INSPIRE].

[70] P.-S. Hsin and A. Turzillo, Symmetry-enriched quantum spin liquids in $(3+1) d$, JHEP 09 (2020) 022 [arXiv: 1904.11550] [INSPIRE].

[71] Y. Hidaka, M. Nitta and R. Yokokura, in preparation.

[72] M. Kawasaki and K. Nakayama, Axions: theory and cosmological role, Ann. Rev. Nucl. Part. Sci. 63 (2013) 69 [arXiv:1301.1123] [INSPIRE].

[73] M. Eto, M. Kurachi and M. Nitta, Constraints on two Higgs doublet models from domain walls, Phys. Lett. B 785 (2018) 447 [arXiv: 1803. 04662] [INSPIRE].

[74] M. Eto, M. Kurachi and M. Nitta, Non-Abelian strings and domain walls in two Higgs doublet models, JHEP 08 (2018) 195 [arXiv: 1805.07015] [INSPIRE].

[75] M. Nitta, Josephson vortices and the Atiyah-Manton construction, Phys. Rev. D 86 (2012) 125004 [arXiv: 1207.6958] [INSPIRE].

[76] M. Kobayashi and M. Nitta, Sine-Gordon kinks on a domain wall ring, Phys. Rev. D 87 (2013) 085003 [arXiv: 1302.0989] [INSPIRE].

[77] R. Shankar, The SO(3) monopole catalog, Phys. Rev. D 14 (1976) 1107 [InSPIRE].

[78] Y. Kawaguchi, M. Nitta and M. Ueda, Knots in a spinor Bose-Einstein condensate, Phys. Rev. Lett. 100 (2008) 180403 [Erratum ibid. 101 (2008) 029902] [arXiv:0802.1968] [INSPIRE].

[79] E. Gerchkovitz and A. Karasik, Vortex-strings in $N=2$ SQCD and bulk-string decoupling, JHEP 02 (2018) 091 [arXiv:1710.02203] [INSPIRE].

[80] E. Gerchkovitz and A. Karasik, New vortex-string worldsheet theories from supersymmetric localization, JHEP 03 (2019) 090 [arXiv:1711.03561] [INSPIRE].

[81] A. Karasik, Vortex-strings in $N=2$ quiver $\times \mathrm{U}(1)$ theories, JHEP 12 (2018) 129 [arXiv: 1808.00725] [INSPIRE]. 